\title{
Distinct Subsets of Syt-IV/BDNF Vesicles Are Sorted to Axons versus Dendrites and Recruited to Synapses by Activity
}

\author{
Camin Dean, ${ }^{1,2,3}$ Huisheng Liu, ${ }^{1,2}$ Thorsten Staudt, ${ }^{4,5}$ Markus A. Stahlberg, ${ }^{3}$ Siv Vingill, ${ }^{3}$ Johanna Bückers, ${ }^{5}$ Dirk Kamin, ${ }^{5}$ \\ Johann Engelhardt, ${ }^{4}$ Meyer B. Jackson, ${ }^{1}$ Stefan W. Hell, ${ }^{4,5}$ and Edwin R. Chapman ${ }^{1,2}$ \\ ${ }^{1}$ Department of Neuroscience and ${ }^{2}$ Howard Hughes Medical Institute, University of Wisconsin, Madison, Wisconsin 53706, ${ }^{3}$ The European Neuroscience \\ Institute Göttingen (ENI-G), D-37077 Göttingen, Germany, ${ }^{4}$ Division of Optical Nanoscopy, German Cancer Research Center (DKFZ) and BioQuant, \\ D-69120 Heidelberg, Germany, and 5Department of NanoBiophotonics, Max Planck Institute for Biophysical Chemistry, D-37077 Göttingen, Germany
}

BDNF plays a critical role in the regulation of synaptic strength and is essential for long-term potentiation, a phenomenon that underlies learning and memory. However, whether BDNF acts in a diffuse manner or is targeted to specific neuronal subcompartments or synaptic sites to affect circuit function remains unknown. Here, using photoactivation of BDNF or syt-IV (a regulator of exocytosis present on BDNF-containing vesicles) in transfected rat hippocampal neurons, we discovered that distinct subsets of BDNF vesicles are targeted to axons versus dendrites and are not shared between these compartments. Moreover, syt-IV-and BDNF-harboring vesicles are recruited to both presynaptic and postsynaptic sites in response to increased neuronal activity. Finally, using syt-IV knockout mouse neurons, we found that syt-IV is necessary for both presynaptic and postsynaptic scaling of synaptic strength in response to changes in network activity. These findings demonstrate that BDNF-containing vesicles can be targeted to specific sites in neurons and suggest that syt-IVregulated BDNF secretion is subject to spatial control to regulate synaptic function in a site-specific manner.

\section{Introduction}

Neurotrophins were initially discovered via their positive effect on neuronal differentiation and survival (Lewin and Barde, 1996). More recently, neurotrophins such as BDNF have been found to modulate synaptic function and plasticity (Poo, 2001); BDNF is essential for long-term potentiation (LTP) (Korte et al., 1995) and for normal learning and memory (Linnarsson et al., 1997, Liu et al., 2004). A critical unresolved issue concerns the synapse specificity of neurotrophin targeting and action (Poo, 2001, Lu et al., 2008). Recent findings raise the hypothesis that neurotrophins might act in a site-specific manner. For example, BDNF-coated beads promote local nerve growth (Tucker et al., 2001) and potentiate presynaptic strength only at nearby synapses (Zhang and Poo, 2002). In addition,

Received Sept. 3, 2011; revised Feb. 17, 2012; accepted Feb. 23, 2012.

Author contributions: C.D., H.L., M.B.J., and E.R.C. designed research; C.D., H.L., T.S., M.A.S., S.V., and D.K. performed research; J.B., J.E., and S.W.H. contributed unpublished reagents/analytic tools; C.D., H.L., T.S., M.A.S., and S.V. analyzed data; C.D. and E.R.C. wrote the paper.

This work was supported by NIH Grant MH61876 to E.R.C., the Epilepsy Foundation, the NIH National Research Service Award, the Sofja Kovalevskaja Award from the Alexander von Humboldt Foundation, and European Research Council grants to C.D. We thank Ulrike Engel and Christian Ackermann at the Nikon Imaging Center at the University of Heidelberg for assistance with photoactivation experiments, Thomas Dresbach for the use of his laboratory while in Heidelberg, Jon Audhya for the use of his NIS Elements software for analysis, Akhil Bhalla for assistance with Western blot experiments, and Mark Dunning for assistance with molecular biology. E.R.C. is an investigator of the Howard Hughes Medical Institute.

This article is freely available online through the J Neurosci Open Choice option.

Correspondence should be addressed to either of the following: Dr. Edwin R. Chapman, Department of Neuroscience, University of Wisconsin, 1300 University Avenue, SMI 129, Madison, WI 53706, E-mail:chapman@wisc.edu; or Dr. Camin Dean, European Neuroscience Institute, Grisebachstrasse 5, Göttingen, Germany D-37077, E-mail: c.dean@eni-g.de.

DOI:10.1523/JNEUROSCI.4515-11.2012

Copyright $\odot 2012$ the authors $\quad 0270-6474 / 12 / 325398-16 \$ 15.00 / 0$
BDNF secreted from BDNF-overexpressing neurons increases dendritic branching only in neighboring neurons (Horch and Katz, 2002), and induced BDNF release from single neurons causes phosphorylation of CREB only in neighboring neurons (Kuczewski et al., 2008). These studies demonstrate local effects of BDNF secretion. However, whether BDNF vesicles themselves are targeted to specific sites for local secretion remains unexplored.

BDNF-containing vesicles have recently been found to harbor the integral membrane protein syt-IV (Dean et al., 2009), a member of the syt family of molecules (Koh and Bellen, 2003, Chapman, 2008) and an immediate early gene (Vician et al., 1995, Ibata et al., 2000) that is important for learning and memory (Ferguson et al., 2000, 2004). This isoform binds SNARE proteins but fails to bind membranes in response to calcium (Chapman et al., 1998, Wang and Chapman, 2010), thereby inhibiting BDNF release from vesicles harboring syt-IV (Dean et al., 2009). In coculture experiments, presynaptic strength is increased only in terminals contacting syt-IV knockout neurons (which have increased BDNF release), and not at nearby wild-type synapses (Dean et al., 2009). This further suggests local effects of BDNF secretion. However, whether neurotrophins are targeted specifically to synaptic sites in response to activity, whereby they could modulate synaptic function via local secretion (Poo, 2001, Lu et al., 2008), remains unknown. Time lapse imaging studies of BDNF vesicles tagged with BDNF-GFP or syt-IV-GFP indicate that these vesicles are highly mobile (Adachi et al., 2005, Dean et al., 2009). Thus, neurons have the potential to dynamically target BDNF vesicles to specific sites along neuronal processes to modify synaptic function locally. 
Here we examined the directed movement and specificity of syt-IV and BDNF vesicle targeting and the effect of syt-IVregulated BDNF release on presynaptic and postsynaptic function in response to alterations in neuronal activity. We discovered that: (1) distinct subsets of syt-IV- and BDNF-harboring vesicles are differentially sorted to axons and dendrites and are not shared between compartments; (2) syt-IV- and BDNF-harboring vesicles are recruited to both presynaptic and postsynaptic sites in response to neuronal activity; and (3) syt-IV is necessary for both presynaptic and postsynaptic scaling of synaptic strength in response to changes in activity. Thus, BDNF secretion regulated by syt-IV is subject to extensive spatial control to regulate synaptic function.

\section{Materials and Methods}

Hippocampal neuron cultures and cocultures. Rat hippocampi were isolated from embryonic day 18-19 rats as described previously (Banker and Cowan, 1977). Syt-IV knockout mice were provided by H. Herschman (University of California, Los Angeles, CA), and hippocampi were isolated from postnatal day 1-3 mice of either sex from syt-IV knockout heterozygote matings, where syt-IV knockout cultures could be directly compared to wild-type littermate cultures. Conditional BDNF knockout mice and CaMKII-Cre mice (provided by Alexei Morozov, $\mathrm{NIH}$, Bethesda, MD) were crossed to generate homozygous forebrainspecific BDNF knockouts, and these mice were crossed to syt-IV knockout heterozygotes for electrophysiology experiments comparing BDNF knockout to BDNF/syt-IV double-knockout littermate hippocampal cultures. Hippocampi were treated with trypsin for $20 \mathrm{~min}$ at $37^{\circ} \mathrm{C}$, triturated to dissociate cells, plated at $25,000-50,000 \mathrm{cells} / \mathrm{cm}^{2}$ on polylysine-coated coverslips (Carolina Biologicals), and cultured in Neurobasal medium supplemented with 2\% B-27 and 2 mm GlutaMAX (Invitrogen Gibco).

For HEK293 cell/neuron cocultures, HEK cells were first cultured alone in DMEM with $10 \%$ fetal bovine serum and transfected using Lipofectamine 2000 (Invitrogen) according to the manufacturer's instructions. After $24 \mathrm{~h}$, these cells were mechanically dissociated in Neurobasal culture medium by gentle trituration and added to 12 days in vitro (DIV) neuronal cultures at a density of $1000-2000 \mathrm{cells} / \mathrm{cm}^{2}$.

All procedures involving animals were performed in accordance with the guidelines of the National Institutes of Health as approved by the Animal Care and Use Committee of the University of Wisconsin-Madison.

Antibodies, reagents, and mammalian expression constructs. Antibodies used were syt-IV, vesicular GABA transporter (VGAT) (Synaptic Systems), VGluT1, GluR2, synapsin, MAP2 (Millipore Bioscience Research Reagents), BDNF (Promega, Sigma), GFP, syt-17 (Abcam), synaptophysin (provided by R. Jahn, Max Planck Institute for Biophysical Chemistry, Göttingen, Germany), PSD-95 (Affinity Bioreagents), and bassoon (Stressgen). Cy2 and Cy3 secondary antibodies were from Jackson ImmunoResearch Laboratories, and Alexa Fluor 647 secondary antibody was from Invitrogen. Chemicals used were forskolin, glycine, TTX, APV (Sigma), and MG132 (Calbiochem). Mammalian expression constructs used were pEGFP-N1 (Clontech), mCherry (provided by R. Tsien, University of California, San Diego, CA), and Nlg-GFP (provided by T. Dresbach, University of Heidelberg, Heidelberg, Germany). Nrx-GFP was made by inserting PCR-amplified GFP into a unique SalI site in vesicular stomatitis virus-tagged $\beta$-NRX-1 just after the LNS domain (constructed by $\mathrm{H}$. Lee and provided by E. Isacoff, University of California, Berkeley, CA). Nlg-IRES-GFP was made by inserting Neuroligin-1 (provided by P. Scheiffele, Biozentrum, University of Basel, Basel, Switzerland) into pIRES2-EGFP (Clontech). BDNF-PAGFP (photoactivatable GFP) was constructed by replacing the GFP in BDNF-GFP described previously (Dean et al., 2009) with PAGFP (provided by J. LippincottSchwartz, NIH, Bethesda, MD). PAGFP-sytIV was constructed by replacing the GFP in previously described GFP-sytIV (Dean et al., 2009) with PAGFP. GFP-syt17 was made by replacing the pHluorin in pHluorin-syt-1 (provided by T. Ryan, Weill Medical College of Cornell
University, New York, NY) with GFP and the syt-1 with syt-17 (provided by M. Craxton, MRC Laboratory, Cambridge, UK).

Transfection of hippocampal neurons. Neurons on $12 \mathrm{~mm}$ coverslips in 24-well plates were transfected with Lipofectamine or calcium phosphate. For Lipofectamine transfection (at 6-9 DIV), medium was removed, saved, and replaced with $500 \mu \mathrm{l}$ of fresh medium. One microliter of Lipofectamine 2000 (Invitrogen) in $50 \mu \mathrm{l}$ of Neurobasal medium and $0.5 \mu \mathrm{g}$ of DNA in 50 $\mu \mathrm{l}$ of Neurobasal medium were incubated separately for $5 \mathrm{~min}$ and then mixed and incubated for $30 \mathrm{~min}$ at room temperature. This mixture was added to the well of neurons, incubated for $4 \mathrm{~h}$ at $37^{\circ} \mathrm{C}$ and $5 \% \mathrm{CO}_{2}$, and then removed and replaced with half saved and half fresh medium.

Neurons were transfected by calcium phosphate at 3-4 DIV as described previously (Dresbach et al., 2003). Before transfection, medium was removed, saved, and replaced with $500 \mu \mathrm{l}$ of $37^{\circ} \mathrm{C}$ Opti-MEM (Invitrogen) and incubated for 30-60 min. One hundred five microliters of transfection buffer (in mM: $274 \mathrm{NaCl}, 10 \mathrm{KCl}, 1.4 \mathrm{Na}_{2} \mathrm{HPO}_{4}, 15$ glucose, and 42 HEPES, pH 7.06) was added dropwise to a solution containing 7 $\mu \mathrm{g}$ of DNA and $250 \mathrm{~mm} \mathrm{CaCl}_{2}$, with gentle vortexing. This mixture was incubated for $20 \mathrm{~min}$ at room temperature, $30 \mu \mathrm{l}$ was added per well, and neurons were incubated for 60-90 min. This medium was then removed, cells were washed $3 \times$ in $37^{\circ} \mathrm{C}$ Neurobasal medium, and saved medium was added back to the transfected cells.

Photoactivation experiments. For photoactivation experiments, coverslips of neurons cotransfected with sytIV-PAGFP/mCherry or with BDNF-PAGFP/mCherry were transferred to a live imaging chamber (Warner Instruments) containing (in mM): $119 \mathrm{NaCl}, 2.5 \mathrm{KCl}, 2 \mathrm{CaCl}_{2}, 2$ $\mathrm{MgCl}_{2}, 30$ glucose, and 25 HEPES, pH 7.4. Images were acquired on a Nikon A1Rsi 32 channel spectral imaging inverted confocal laser microscope equipped with a Perfect Focus System using $40 \times$ or $60 \times$ objectives and a hybrid galvano/resonant scanner for simultaneous photoactivation and imaging (at the Nikon Imaging Center of the University of Heidelberg, Heidelberg, Germany).

DIV 6-8 transfected cells were visualized by mCherry fluorescence and chosen such that axons and dendrites could be clearly identified based on morphology (and verified by retrospective immunostaining for MAP2 to mark dendrites in all cases where axons and dendrites were difficult to distinguish). For each time lapse experiment, a baseline of GFP and mCherry fluorescence was collected for $10 \mathrm{~s}$ before photoactivation of the region of interest with $405 \mathrm{~nm}$ laser light. Regions to photoactivate in axons, dendrites, or cell bodies were chosen to include the largest region possible in the compartment of interest without including any other regions. Figure $1 \mathrm{~A}$ shows the specificity of photoactivation within defined regions. Regions were photoactivated continuously with the $405 \mathrm{~nm}$ laser at $40 \%$ laser power and a pixel dwell time of $20 \mu$ s during simultaneous acquisition of GFP and mCherry fluorescence, such that all vesicles transiting through the region during the time lapse were photoactivated. Laser intensity and pixel dwell time were selected to maximize photoactivation and minimize bleaching. The mCherry channel was imaged simultaneously and served as a control for focal drift.

Following image collection, cell bodies, axons, and dendrites were selected as regions in NIS Elements software and fluorescence intensity was plotted versus time. Average fluorescence intensity at the end of the time lapse was calculated in $10 \mu \mathrm{m}$ long $\times 3 \mu \mathrm{m}$ wide regions of processes in axons and dendrites proximal to the cell body. For axon photoactivation experiments, fluorescence intensity was calculated in a region within the photo-activated area, in an area outside and just distal to the photoactivated area, in the cell body, and in proximal dendrites at $t=0$ and $10 \mathrm{~min}$. For dendrite photoactivation, fluorescence intensity was calculated in a region within the photoactivated area, in a different dendrite (i.e., the vesicles would have to travel from the photoactivated dendrite into the cell body and then into another dendrite), in the cell body, and in the proximal region of the axon, at $t=0$ and $10 \mathrm{~min}$.

Immunocytochemistry, image acquisition, and quantitation. Cells were fixed with $4 \%$ paraformaldehyde, permeabilized, blocked in $10 \%$ goat serum and $0.1 \%$ Triton X-100, and immunostained with primary antibodies at room temperature for $4 \mathrm{~h}$ or overnight at $4^{\circ} \mathrm{C}$.

Images of immunostained cells were acquired on an Olympus FV1000 upright confocal microscope with a $60 \times 1.10$ numerical aperture waterimmersion lens. Lasers with wavelengths of 488, 543, and $635 \mathrm{~nm}$ were 


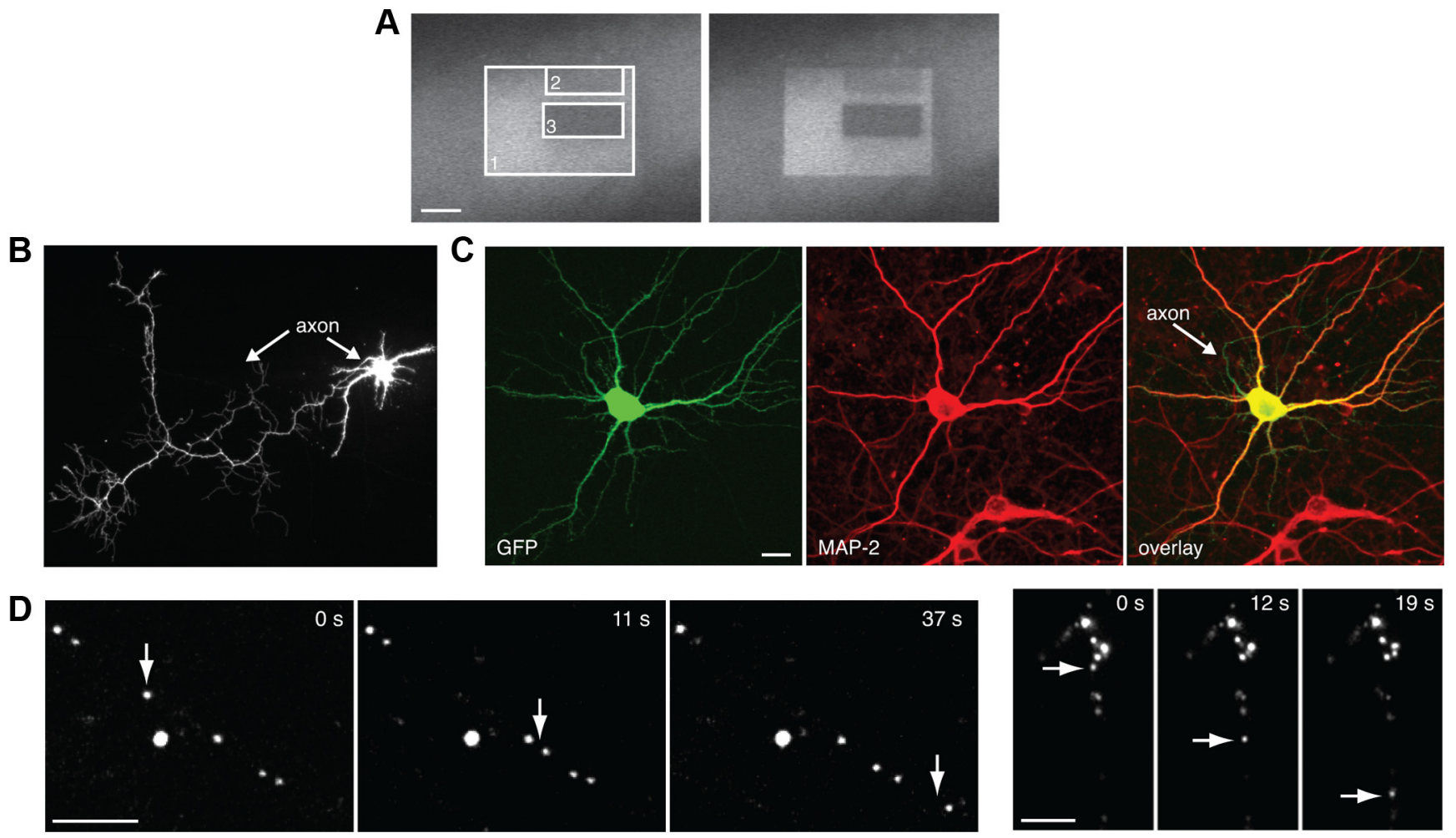

Figure 1. Spatial accuracy of photoactivation and identification of axons versus dendrites. A, A slide streaked with photoactivatable fluorescein was photoactivated with $40 \%$ laser power $405 \mathrm{~nm}$ light in rectangular ROI 1, followed by bleaching with $10 \% 488 \mathrm{~nm}$ laser power in ROI 2, and $40 \% 488 \mathrm{~nm}$ laser power in ROI 3 . Scale bar is $5 \mu \mathrm{m}$. B, C, Verification of axons versus dendrites in a low-magnification image of a GFP-transfected neuron where the axon can be traced back to the cell body and identified in higher-magnification imaging experiments $(\boldsymbol{B})$ and by retrospective immunostaining with the dendritic marker MAP-2 (C). Scale bar is $10 \mu \mathrm{m}$.D. Time lapse images of trafficking GFP-tagged BDNFvesides imaged by confocal (left panels) and epifluorescence (right panels). Scale bar is $3 \mu$ m in left panels and $5 \mu$ m in right panels.

used, with Olympus FV1000 automated dichroic/emission settings for sequential scanning of Cy2, Cy3, and Alexa Fluor 647 secondary dyes. For quantitation, images were acquired with identical laser and gain settings and imported into MetaMorph (Improvision) for analysis. For percentage colocalization of syt-IV at synapses (Fig. 1), thresholds were chosen for the VGluT channel (green), VGAT channel (blue), and syt-IV channel (red), such that all recognizable puncta were selected. Since the syt-IV antibody cross-reacts with other proteins in Western blots of tissue isolated from brain, we used knockout mice as a control for specificity. Confocal gain in all experiments was set such that no syt-IV signal was visible in syt-IV knockout neurons. The average intensity of syt-IV signal within VGluT or VGAT puncta was determined using MetaMorph for all conditions and expressed as percentage of control. For percentage colocalization, the fraction of syt-IV-positive signal within VGluT- or VGATpositive puncta was calculated using MetaMorph. Colocalization between VGluT and VGAT was 5\%, and a positive control double immunostain using an antibody against the luminal domain of syt I and another antibody against the cytoplasmic domain of syt I showed $90 \%$ overlap. Cell bodies and regions $10 \mu \mathrm{m}$ proximal to cell bodies were excluded from analysis because the syt-IV signal in the soma often overlapped with inhibitory terminals on cell bodies.

For quantitation of immunofluorescence signals on Nlg-IRES-GFP-, Nlg-GFP-, Nrx-GFP-, or GFP-expressing HEK293 cells, transfected HEK cells were marked by thresholding the GFP signal. The average intensity of syt-IV signal within this thresholded region was then determined. Statistical significance for all quantitation was determined by a Student's $t$ test where ${ }^{\star} p<0.05,{ }^{* *} p<0.01$, and ${ }^{* * *} p<0.001$.

Protein purification and Western blots. Twelve to fourteen DIV hippocampal neurons treated with $200 \mu \mathrm{M}$ glycine for $15 \mathrm{~min}$ or $1 \mathrm{~h}, 50 \mu \mathrm{M}$ forskolin for $4 \mathrm{~h}, 50 \mu \mathrm{M}$ MG132 for $4 \mathrm{~h}, 1 \mu \mathrm{M}$ TTX for $48 \mathrm{~h}$, or in control conditions growing in $10 \mathrm{~cm}$ dishes at a density of $50,000 \mathrm{cells} / \mathrm{cm}^{2}$, were washed twice in PBS and $1 \mathrm{ml}$ of hypotonic buffer $(10 \mathrm{~mm}$ Tris- $\mathrm{HCl}, \mathrm{pH}$ 7.4) was added per plate for $5 \mathrm{~min}$. Cells were harvested in $0.5 \mathrm{ml}$ of homogenization buffer (in mm: 320 sucrose, 1 EDTA, 10 Tris- $\mathrm{HCl}, \mathrm{pH}$
7.4) by scraping and passed through a 27-gauge needle 10 times. A final concentration of $150 \mathrm{~mm} \mathrm{NaCl}$ was added to the lysate. Cell lysates were centrifuged at $4000 \mathrm{rpm}$ for $10 \mathrm{~min}$ to pellet nuclei and cellular debris. The supernatant was collected and the protein concentration determined by BCA assay. Twelve micrograms of total protein per lane was resolved by SDS-PAGE and analyzed by immunoblotting for syt-IV (which runs as a $46 \mathrm{kDa}$ band).

Stimulated emission depletion microscopy. For imaging of syt-IV/synaptophysin or syt-I/ synaptophysin, hippocampal neurons were fixed and immunostained with Atto565 and Dy-485XL secondary antibodies (Dianova). Pulsed excitation of Atto565 (Atto-Tec) was achieved using a high repetition rate laser diode source (PicoTA, PicoQuant) at a wavelength of $\lambda_{\text {exc }}=532 \mathrm{~nm}$ (where exc is excitation), synchronized with the stimulated emission depletion (STED) microscopy laser via a photodiode (Alphalas). Dy-485XL (Dyomics) was excited by the $470 \mathrm{~nm}$ line from a pulsed high-repetition rate laser diode source (Picoquant). Fluorescence inhibition was accomplished at $\lambda_{\mathrm{STED}}=647 \mathrm{~nm}$ using an actively mode locked ArKr laser (Spectra Physics). The excitation and modulation beams were combined using acousto-optical tunable filters (AOTFs) (Crystal Technology) and coupled into a microscope stand (DMI 4000B, Leica Microsystems) equipped with a three-axis piezo stage scanner (Physik Instrumente). The AOTFs enabled blanking of the lasers and allowed the power of each laser beam to be controlled independently. They also provided a means for selecting counter-propagating fluorescence returning from the confocal microscope. The collected fluorescence passed through an additional bandpass filter (580/40 for Atto565 and Dy-485XL, AHF Analysentechnik) and was detected confocally with a photon-counting module (SPCM-AQR-13-FC, PerkinElmer). The scanner fly backs were blanked via the line signal from the data acquisition software (Imspector, MPI). A vortex phase plate (RPC Photonics) was used in the STED beam path to generate a donut-shaped light distribution in the focal plane.

BDNF and Syt-IV were immunostained in fixed hippocampal neurons using Atto590 and Atto647N secondary antibodies (Dianova) and im- 

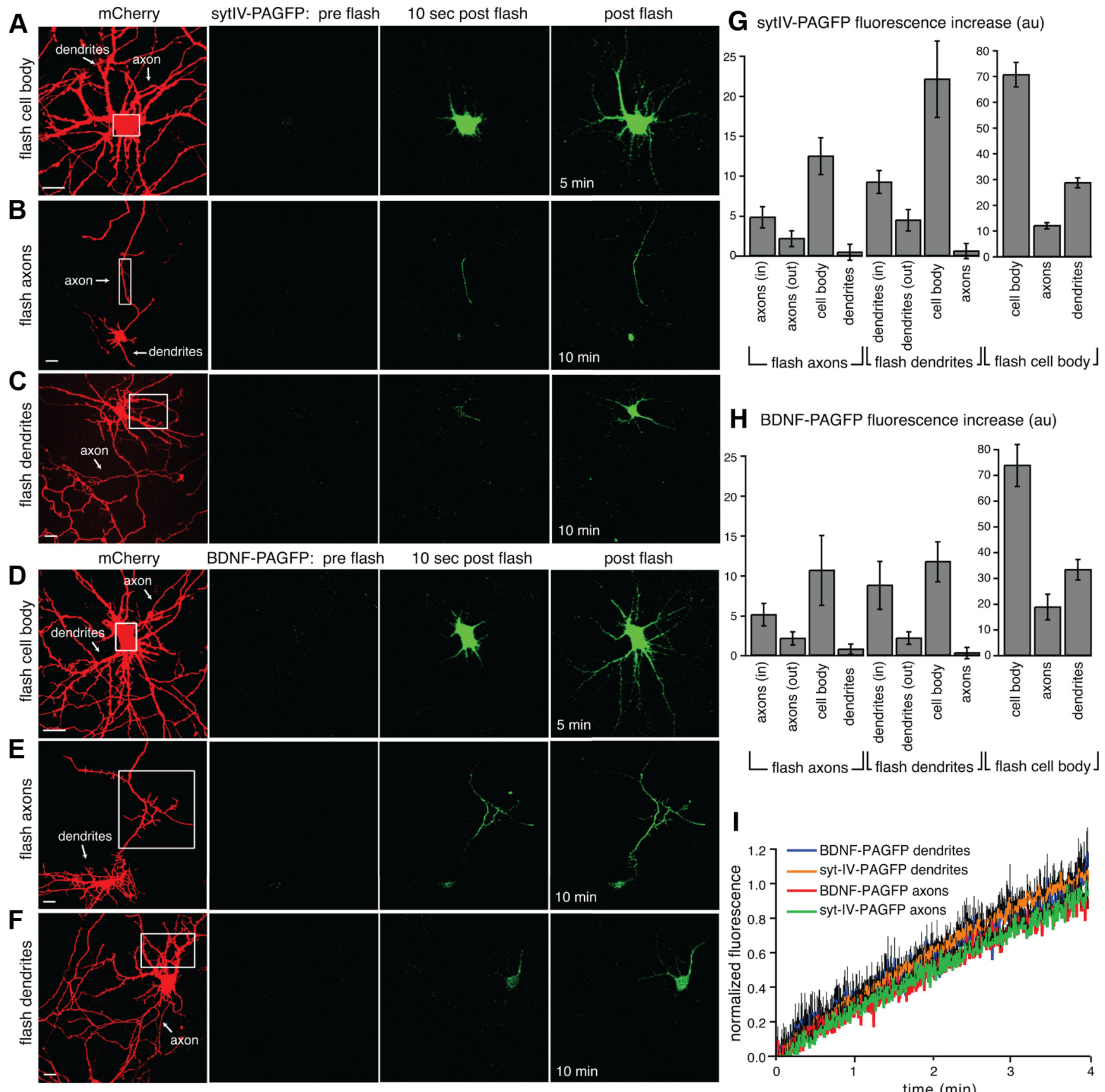

L flash axons $\left.\lrcorner \bigsqcup_{\text {flash dendrites }}\right\rfloor$ flash cell body $\rfloor$

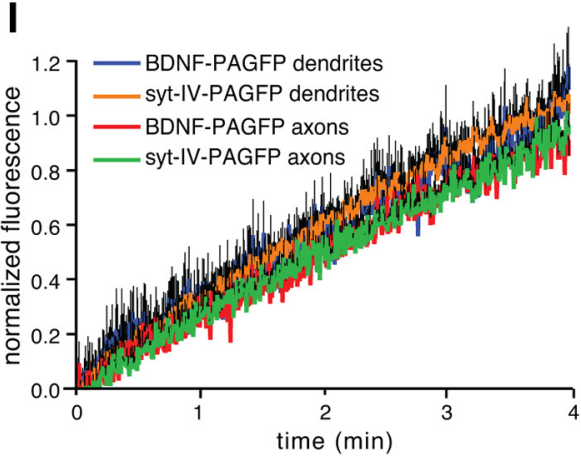

Figure 2. Syt-IV-and BDNF-harboring vesicles are not shared between axons and dendrites. $\boldsymbol{A}-\boldsymbol{C}$, Images of a hippocampal neuron cotransfected with sytIV-PAGFP and $m$ Cherry before and after photoactivation of the cell body $(\boldsymbol{A})$, axon $(\boldsymbol{B})$, or dendrites $(\boldsymbol{C})$ in the indicated regions. $\boldsymbol{D}-\boldsymbol{E}$, Neuron cotransfected with BDNF-PAGFP/mCherry before and after photoactivation of the cell body (D), axon $(\boldsymbol{E})$, or dendrites $(\boldsymbol{F})$. $\boldsymbol{G}$, Quantitation of fluorescence increase in the cell body, dendrites, and axons of sytlV-PAGFP-transfected neurons following photoactivation in the indicated regions. For axon photoactivation experiments, fluorescence intensity was calculated in a region within (in) the photoactivated area, in an area outside (out) just distal to the photoactivated area, in the cell body, and in proximal dendrites. For dendrites, fluorescence intensity was calculated in a region within (in) the photoactivated area, in a different dendrite (where the vesicles would have to travel from the photoactivated dendrite, into the cell body, and then into another dendrite) designated "dendrite (out)," in the cell body, and in the proximal region of the axon. $\boldsymbol{H}$, Quantitation of fluorescence in the cell body, dendrites, and axons of BDNF-PAGFP-transfected neurons following photoactivation. $I$, Time course of fluorescence increase in cell bodies following photoactivation of axons or dendrites ( $n=12$ time lapse experiments for each condition; 3 cultures/transfections; error bars indicate SEM). Scale bars are $10 \mu \mathrm{m}$ in all panels.

aged with a custom-built two-color STED microscope as described previously (Bückers et al., 2011). The excitation wavelengths $\lambda_{\text {exc,Atto590 }}=$ $570 \mathrm{~nm}$ and $\lambda_{\text {exc,Atto647N }}=650 \mathrm{~nm}$ and the STED wavelengths $\lambda_{\mathrm{STED}, \mathrm{Atto590}}=720 \pm 10 \mathrm{~nm}$ and $\lambda_{\mathrm{STED}, \text { Atto647N }}=755 \pm 15 \mathrm{~nm}$ were all provided by a single supercontiuum laser source (SC-450-PP-HE, Fianium). Fluorescence was detected with two separate confocal detection units covering the spectral ranges of $\lambda_{\text {fluo,Atto590 }}=620 \pm 20 \mathrm{~nm}$ and $\lambda_{\text {flluo,Atto647N }}=670 \pm 15 \mathrm{~nm}$. The application of a pulse-interleaved acquisition scheme enabled the simultaneous recording of both imaging channels. No relative shift of the images, i.e., due to a drift of the sample, is expected. In addition, crosstalk, i.e., leakage of signal into the wrong imaging channel, was eliminated by linear unmixing.

Images were preprocessed using a single-step linear deconvolution, i.e., Wiener filter, which was carried out with a theoretical effective point spread function of $40 \mathrm{~nm}$ FWHM.

Electrophysiology. Whole-cell patch-clamp recordings of mEPSCs were made using 15 to 21 DIV dissociated hippocampal cultures from syt-IV knockout and wild-type littermates (in control conditions and following 

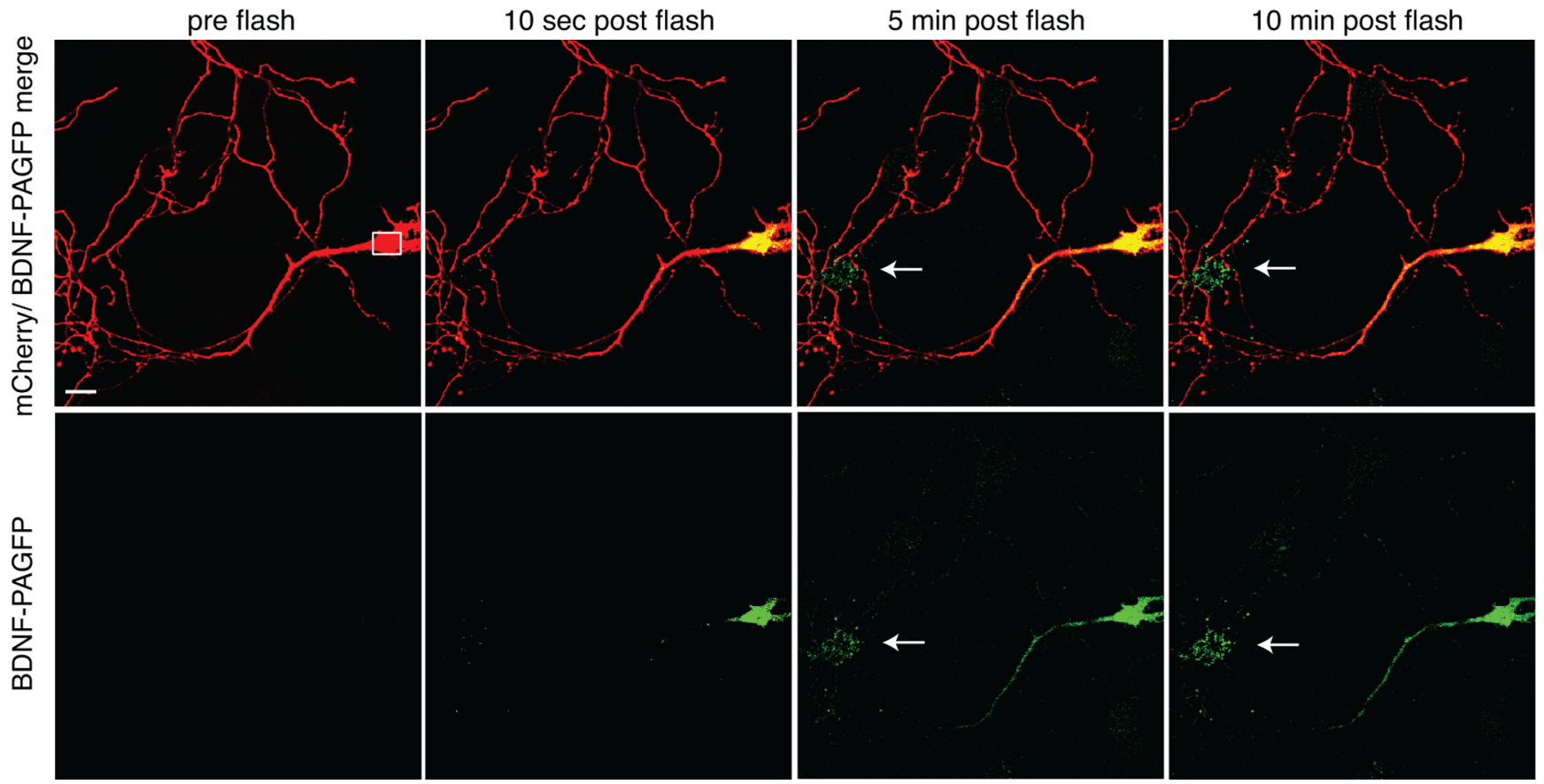

Figure 3. BDNF-PAGFP transfer to neighboring cells. BDNF-PAGFP is secreted and taken up by neighboring neurons; photoactivation of the cell body of a hippocampal neuron cotransfected with $\mathrm{mCherry} \mathrm{and} \mathrm{BDNF-PAGFP} \mathrm{is} \mathrm{shown.} \mathrm{Note} \mathrm{that} \mathrm{photoactivated} \mathrm{BDNF-PAGFP} \mathrm{is} \mathrm{taken} \mathrm{up} \mathrm{by} \mathrm{the} \mathrm{soma} \mathrm{of} \mathrm{a} \mathrm{neighboring} \mathrm{cell} \mathrm{(arrows).} \mathrm{Scale} \mathrm{bar} \mathrm{is} 10 \mu \mathrm{m}$.

treatment with $1 \mu \mathrm{M}$ TTX or $200 \mu \mathrm{M}$ glycine $/ 10 \mu \mathrm{M}$ bicuculline for $48 \mathrm{~h}$ or BDNF knockout and syt-IV/BDNF double knockout littermate cultures. Neurons were voltage clamped at $-70 \mathrm{mV}$ in the presence of $1 \mu \mathrm{M} \mathrm{TTX}$, and currents were recorded using an EPC-10/2 amplifier (HEKA Electronik) and Patchmaster software (HEKA). Cells were continuously perfused with extracellular solution consisting of, in mM: $140 \mathrm{NaCl}, 5 \mathrm{KCl}$, $1.25 \mathrm{NaH}_{2} \mathrm{PO}_{4}, 26 \mathrm{NaHCO}_{3}, 2 \mathrm{CaCl}_{2}, 1 \mathrm{MgCl}_{2}, 10$ glucose, and $50 \mathrm{APV}$ bubbled with $95 \% \mathrm{O}_{2}$ and $5 \% \mathrm{CO}_{2}$ (pH 7.3, $\left.300 \mathrm{mOsm}\right)$. Intracellular solution consisted of, in mM: $130 \mathrm{~K}$-gluconate, 10 EGTA, 10 HEPES, 5 phosphocreatine, $2 \mathrm{Mg}$-ATP, and 0.3 Na-GTP (pH 7.3, $300 \mathrm{mOsm}$ ). For recording mEPSCs, $0.1 \mathrm{~mm}$ picrotoxin and $1 \mu \mathrm{M}$ TTX were added to the bath solution. For recording mIPSCs, picrotoxin was replaced with 20 $\mu \mathrm{M}$ CNQX. Recordings were filtered at $2.9 \mathrm{kHz}$ and digitized at $5 \mathrm{kHz}$. Events were detected with MiniAnalysis software (Synaptosoft) using a threshold of five times the RMS noise. Statistical significance was determined by a Student's $t$ test where ${ }^{\star} p<0.05,{ }^{* *} p<0.01$, and ${ }^{* * *} p<0.001$.

$F M$ dye destaining experiments. For FM dye experiments, wild-type and syt-IV knockout mouse neuron cultures in control conditions or treated with $1 \mu \mathrm{M}$ TTX for $48 \mathrm{~h}$ were loaded with $10 \mu \mathrm{M}$ FM1-43 in depolarizing buffer (in mM: $100 \mathrm{NaCl}, 45 \mathrm{KCl}, 2 \mathrm{CaCl}_{2}, 2 \mathrm{MgCl}_{2}, 5.5$ glucose, and 20 HEPES, pH 7.3) for 2 min to maximally load all vesicles. Coverslips were then transferred to a $5 \mathrm{~mm} \mathrm{KCl}$ solution (in mM: $140 \mathrm{NaCl}, 5 \mathrm{KCl}, 2 \mathrm{CaCl}_{2}$, $2 \mathrm{MgCl}_{2}, 5.5$ glucose, and 20 HEPES, ph 7.3) for $5 \mathrm{~min}$ to remove excess dye. Coverslips were then mounted in live imaging chambers (Warner Instruments) in the $5 \mathrm{~mm} \mathrm{KCl}$ solution. $\mathrm{FM}$ dye-labeled boutons were visualized with a $484 \mathrm{~nm}$ excitation/605 nm emission filter, and images were acquired on a Nikon TE300 inverted microscope with a Roper Scientific Photometric Cascade II EMCCD camera and a Lambda DG-4 high speed wavelength switcher interfaced with MetaMorph software. Images were collected at $1 \mathrm{~s}$ intervals with $200 \mathrm{~ms}$ exposure times. A baseline was collected for 10 images before addition of depolarizing buffer $(45 \mathrm{~mm}$ $\mathrm{KCl}$ ) to destain neurons. Following image collection, FM dye-labeled boutons were selected as regions in MetaMorph, and fluorescence intensity was plotted versus time. Destain traces were normalized by setting maximal load to one and complete destain (disappearance of bouton to background levels) to zero for comparison of rates. Rate of destain (tau) was determined by a single exponential fit of normalized destain curves.

Voltage imaging. Detection of epileptiform response by voltage imaging was performed as described previously (Chang et al., 2007). Five- week old mice were euthanized with $\mathrm{CO}_{2}$. Brains were removed into and ice-cold cutting solution consisting of, in mM: $124 \mathrm{NaCl}, 3.2 \mathrm{KCl}, 26$ $\mathrm{NaHCO}_{3}, 1.25 \mathrm{NaH}_{2} \mathrm{PO}_{4}, 0.1 \mathrm{CaCl}_{2}, 6 \mathrm{MgSO}_{4}$, and 10 glucose and bubbled with 95\% $\mathrm{O}_{2} / 5 \% \mathrm{CO}_{2}$. Horizontal $350-\mu$ m-thick hippocampal slices were cut with a tissue slicer (HR2, Sigmann Elektronik). Slices were incubated in bubbled artificial cerebrospinal fluid (ACSF), identical to the cutting solution but containing $2.5 \mathrm{mM} \mathrm{CaCl}_{2}$ and $1.3 \mathrm{mM} \mathrm{MgSO}_{4}$, for $1 \mathrm{~h}$, stained with bubbled ACSF containing $0.02 \mathrm{mg} / \mathrm{ml}$ voltage-sensitive absorbance dye RH482 (NK3630, Hayashibara Biochemical Laboratories) for $1 \mathrm{~h}$, and then returned to ACSF. During recording, slices were continuously perfused with bubbled ACSF at $29-31^{\circ} \mathrm{C}$. Slices were stimulated with $200 \mu$ s current pulses delivered by a stimulus isolator (Model A365, World Precision Instruments). The photodiode array instrumentation and software have been described previously (Chang and Jackson, 2006). Optical signals (average of four consecutive trials) are presented as the change in transmitted light divided by the resting light intensity $(\Delta I / I)$ and are proportional to the membrane potential change within the physiological range of voltages.

\section{Results}

Distinct subtypes of syt-IV- and BDNF-harboring vesicles are targeted to axons versus dendrites

Syt-IV- and BDNF-harboring vesicles in axons versus dendrites exhibit distinct intravesicular $\mathrm{pH}$ and different modes of BDNF release (Adachi et al., 2005, Dean et al., 2009, Matsuda et al., 2009). These differences might be dictated by the cellular environment in these two compartments or might be due to specific sorting of distinct subtypes of syt-IV- and BDNF-harboring vesicles to either axons or dendrites. To address this issue, we performed photoactivation experiments using hippocampal neurons. Neurons were cotransfected with either sytIV-PAGFP/ $\mathrm{mCherry}$ or BDNF-PAGFP/mCherry, where the mCherry allows visualization of transfected cells before photoactivation. The PAGFP tagged to syt-IV or BDNF is nonfluorescent until photoactivated by $405 \mathrm{~nm}$ light, which causes it to emit green fluorescence when excited at $488 \mathrm{~nm}$. This approach allows photoactivation of specific subcellular compartments, i.e., axons, 
A

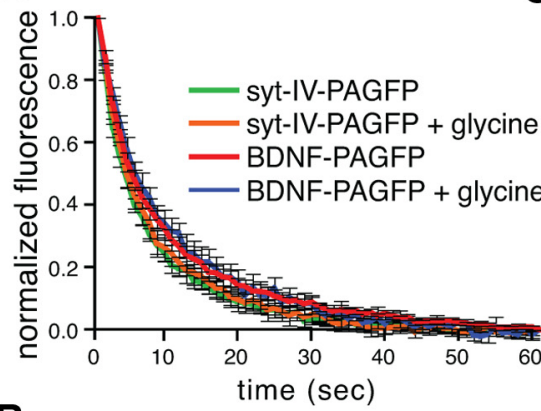

B
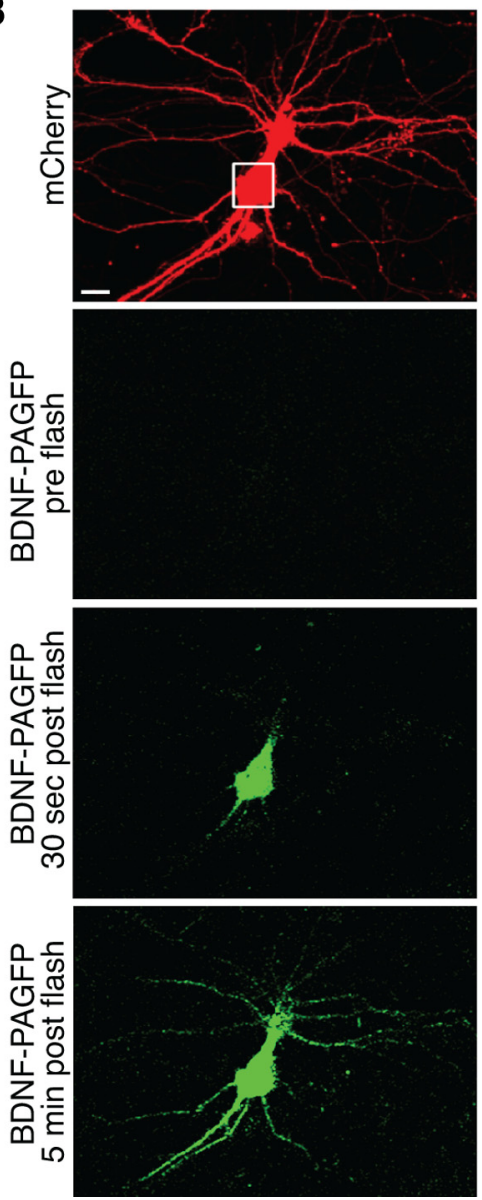

C

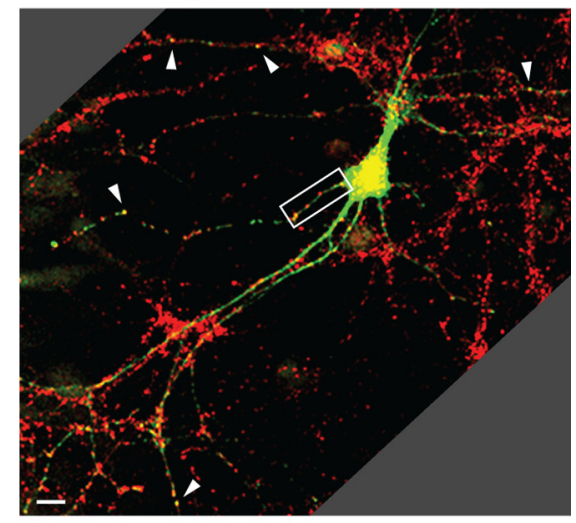

D
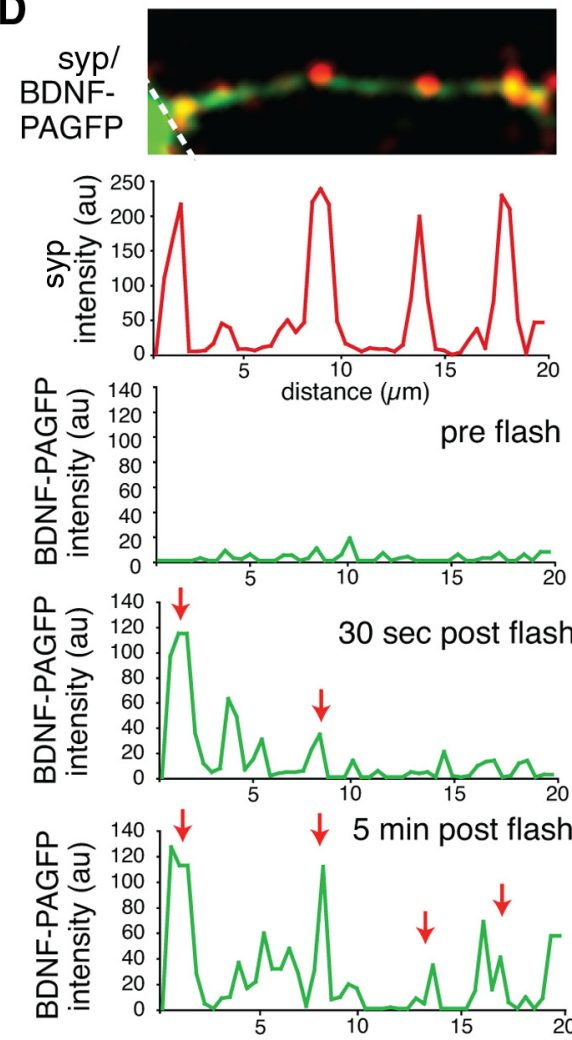

Figure 4. BDNF-PAGFP trafficked from the cell body accumulates at synaptic sites following increased neuronal activity. $\boldsymbol{A}$, Time course of fluorescence decrease in cell bodies (indicating transport of BDNF-PAGFP or syt-IV-PAGFP out of the cell body and into neuronal processes) immediately following photoactivation of the cell body in the presence or absence of $200 \mu \mathrm{m}$ glycine. Tau values of single exponential fits of the data for the indicated condition are: syt-IV-PAGFP $=7.3 \pm 0.7$, syt-IV-PAGFP + glycine $=$ $8.0 \pm 0.9$, BDNF-PAGFP $=8.2 \pm 1.0$, and BDNF-PAGFP + glycine $=8.1 \pm 1.0 . B$, Representative images of a hippocampal neuron cotransfected with BDNF-PAGFP and mCherry and treated with $200 \mu \mathrm{m}$ glycine for 15 min before and throughout and imaging before and after photoactivation of the cell body by illumination of the indicated region. $\boldsymbol{C}$, Retrospective immunostain of the same neuron with an anti-synaptophysin antibody (using a Cy 5 secondary antibody, pseudocolored red) to mark synaptic sites. BDNF-PAGFP accumulates at synaptic sites (arrowheads). D, Time course of accumulation of BDNF-PAGFP at synapses (arrows) along the indicated neuronal process following photoactivation of the cell body. Line scans of intensity of synaptophysin (red line) and BDNF-PAGFP (green lines) along the neuronal process are shown. Dashed line indicates the border of the photoactivated region. Scale bar is $10 \mu \mathrm{m}$.

dendrites, or the soma, and subsequent tracking of the fate of vesicles, visualized as green fluorescence, present in each of these locations.

We first tested the spatial accuracy of photoactivation (Fig. $1 A)$ and two different methods for identification of axons versus dendrites in photoactivation experiments (Fig. $1 B, C$ ): in the majority of cases, axons versus dendrites could clearly be identified

using prior low-magnification imaging where axons extended long distances compared to dendrites (Fig. $1 B$ ). In cases where the two types of processes were difficult to distinguish morphologically, retrospective immunostaining for MAP-2, to mark dendrites, was used (Fig. $1 C$ ). We also verified, using high-magnification of isolated processes where single puncta were clearly visible, that photoactivated (BDNFPAGFP) and non-photoactivated (BDNFGFP) vesicles (Fig. 1D) had similar velocities $(0.60 \pm 0.21 \mu \mathrm{m} / \mathrm{s}$ and $0.63 \pm 0.24$ $\mu \mathrm{m} / \mathrm{s}$, respectively; $n=21$ ) and traveled in both anterograde and retrograde directions $(52.0 \pm 4 \%$ anterograde and $48 \pm 4 \%$ retrograde), indicating that photoactivation did not reduce vesicle mobility.

We then photoactivated the cell bodies of neurons cotransfected with sytIVPAGFP and mCherry by continuous scanning of the cell body at $405 \mathrm{~nm}$ while simultaneously imaging GFP fluorescence with $488 \mathrm{~nm}$ excitation. We found that sytIV-PAGFP vesicles trafficked rapidly out of the soma into both axons and dendrites, reaching distal processes within 5 min of activation (Fig. $2 A, G$ ). Cytoplasmic PAGFP, activated in the cell body, diffused to distal processes much more quickly at a rate of $\sim 35 \pm 3 \mu \mathrm{m}^{2} / \mathrm{sec}$, as reported previously (Bloodgood and Sabatini, 2005). Thus, the translocation of sytIV-PAGFP likely occurs via directed movement of vesicles to which it is attached and not via diffusion of cytoplasmic sytIV-PAGFP. We then specifically photoactivated regions of axons in sytIVPAGFP/mCherry-transfected cells by continuous scanning using pixel dwell times optimized for maximal activation and minimal bleaching to photoactivate all vesicles transiting through the region during the $10 \mathrm{~min}$ period of image acquisition. Following the time lapse, the fluorescence intensity in the soma, dendrites, and axonal regions both within and outside of the photoactivated region were quantified (Fig. 2G). We found that the vesicles within axons traveled from the photoactivated region both away from and toward the cell body (where a significant increase in fluorescence was observed). Surprisingly, despite trafficking back to the cell body within minutes, these vesicles did not enter dendrites to an appreciable extent over tens of minutes (Fig. $2 B, G$ ). We next performed the converse experiment and selectively photoactivated vesicles present in dendrites and followed their trafficking postactivation. In this case, we analyzed the fluorescence within the photoactivated dendrites, within the cell body, in the axon, and within other dendrites that were not photoactivated (i.e., if vesicles trafficked to other dendrites they would have to move from 
A

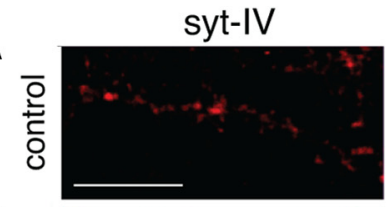

B

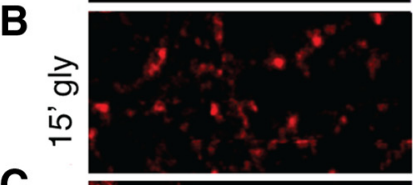

C

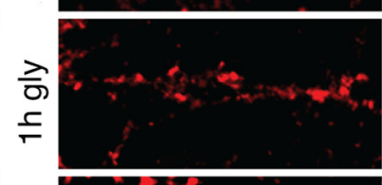

D

E

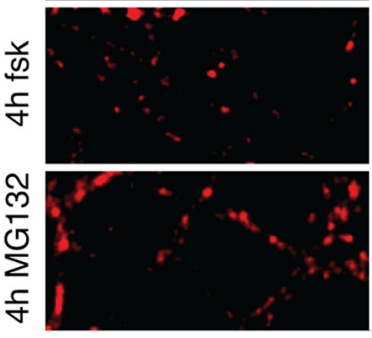

VGAT
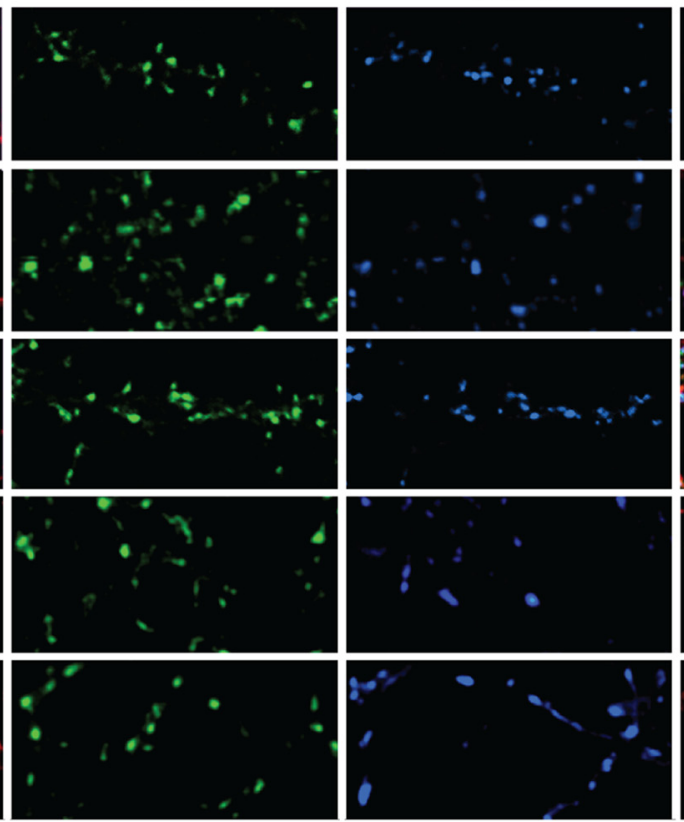
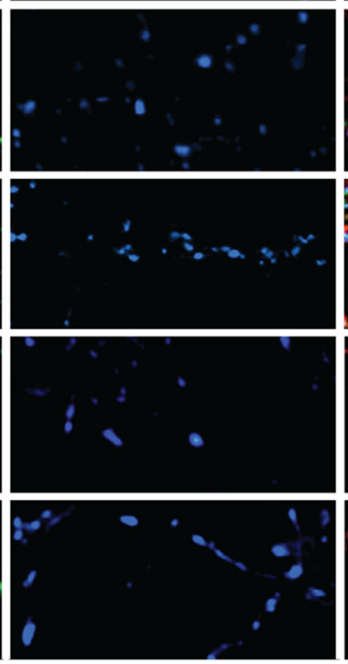

merge
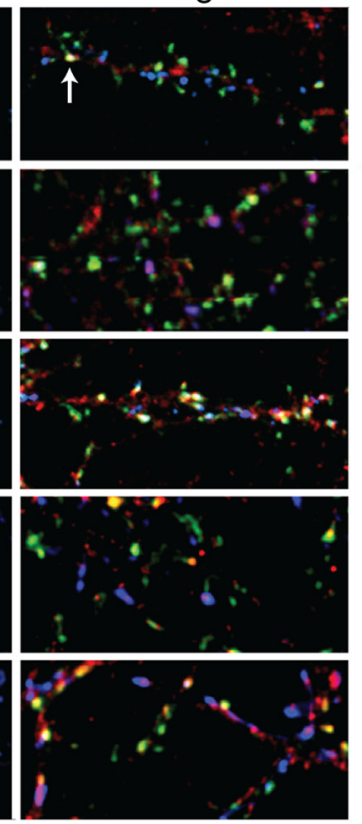

$\mathbf{F}$
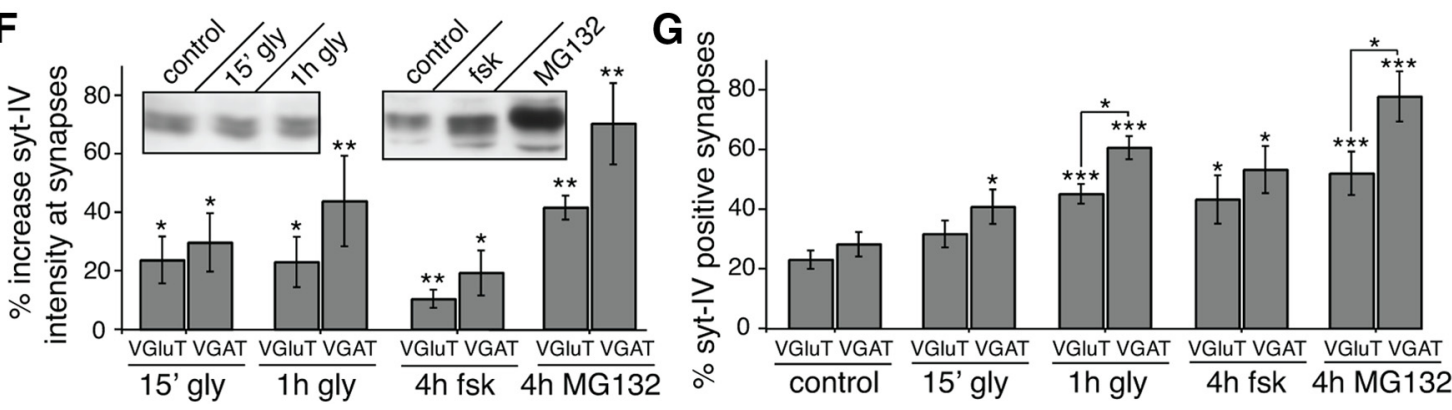

Figure 5. Syt-IV is recruited to synapses by activity. $\boldsymbol{A}-\boldsymbol{E}$, Immunostains of syt-IV and VGluT and VGAT (to mark excitatory and inhibitory synapses, respectively) in hippocampal neurons in control conditions (A) and following treatment with $200 \mu \mathrm{m}$ glycine (gly) for $15 \mathrm{~min}\left(15^{\prime}\right)(\boldsymbol{B})$ or $1 \mathrm{~h}(\boldsymbol{C}), 50 \mu \mathrm{m}$ forskolin (fsk) for $4 \mathrm{~h}(\boldsymbol{D})$, or $50 \mu \mathrm{m} \mathrm{MG132} \mathrm{for} 4 \mathrm{~h}$ (E). $\boldsymbol{F}$, Quantitation of increase in syt-IV average intensity within thresholded VGluT and VGAT puncta expressed as percent of control. Pearson's $r$ for syt-IV/VGluT $=0.10 \pm 0.03$ (control), $0.27 \pm 0.02$ (15' gly), $0.47 \pm 0.08$ ( $1 \mathrm{~h} \mathrm{gly),}$ $0.42 \pm 0.07$ (fsk), and for syt-IV/VGAT $=0.16 \pm 0.07$ (control), $0.37 \pm 0.09$ ( $15^{\prime}$ gly), $0.41 \pm 0.08$ ( $1 \mathrm{~h} \mathrm{gly),} 0.09 \pm 0.21$ (fsk) $(n=10$ images; 2 coverslips each from 5 cultures; error bars indicate SEM). Insets: Western blot of syt-IV in control and treated cultures; equal amounts of protein $(12 \mu \mathrm{g})$ were loaded per lane. $G$, Quantitation of the fraction of syt-IV-positive synapses. VGluT and VGAT puncta were thresholded and the fraction of synapses with detectable syt-IV signal within VGlut or VGAT-positive puncta was calculated for each condition ( $n=10 \mathrm{images} ; 2$ coverslips each from 5 cultures; error bars indicate SEM. Significance was determined by a Student's $t$ test; ${ }^{*} p<0.05$, ${ }^{* *} p<0.01$, and ${ }^{* * *} p<0.001$. Significance relative to control is shown except for indicated comparisons between VGluT and VGAT). Scale bars are $10 \mu \mathrm{m}$ in all panels.

the photoactivated dendrite back through the cell body and into the second dendrite). We found that the vesicles in dendrites were in fact transported to the cell body and between different dendrites, but were excluded from axons (Fig. 2C,G).

We also performed similar photoactivation experiments on cells transfected with BDNF-PAGFP/mCherry. Like sytIVPAGFP, photoactivated BDNF-PAGFP trafficked rapidly into axons and dendrites from the cell body (Fig. 2D, $H$ ). BDNF-PAGFP vesicles in photoactivated axons trafficked anterogradely and retrogradely back to the cell body but could not enter dendrites to a significant extent (Fig. 2E,H). Likewise, the BDNF-PAGFP in photoactivated dendrites could traffic back to the cell body and into other dendrites but failed to enter axons (Fig. $2 F, H$ ). We observed a constant increase in fluorescence in the cell body following photoactivation of axons or dendrites (Fig. 2I), indicating that degradation within the cell body is not a significant parameter to prevent movement of vesicles between compartments on the timescale of our experiments.

We note that more sytIV-PAGFP than BDNF-PAGFP was trafficked back to the cell body following photoactivation of ax- ons or dendrites. This could be a result of release of some of the BDNF-PAGFP from axons or dendrites before or during retrograde transport; we occasionally observed an increase in BDNFPAGFP fluorescence in neighboring cells, indicating that the BDNF-PAGFP can be released and taken up by other neurons, as reported previously (Kohara et al., 2001) (Fig. 3).

\section{Activity-inducing stimuli drive syt-IV/ BDNF vesicles to synapses}

BDNF, and the regulation of its release by syt-IV (Dean et al., 2009), is important for modulating synaptic function during LTP (Poo, 2001). We therefore next tested whether syt-IV and BDNF vesicle trafficking is affected following incubation with $200 \mu \mathrm{M}$ glycine for $15 \mathrm{~min}$ to activate NMDA receptors (Musleh et al., 1997, Lu et al., 2001). Incubation with glycine did not alter the rate of export of syt-IV or BDNF vesicles out of the cell body (Fig. $4 \mathrm{~A}$ ) or the speed of the fluorescence wave (corresponding to multiple vesicles) leaving the cell body; speed (axons) $=1.6 \pm 0.2$ (syt-IV), $1.5 \pm 0.3$ (syt-IV + glycine), $1.5 \pm 0.2(\mathrm{BDNF}), 1.7 \pm$ 0.3 (BDNF + glycine); speed (dendrites) $=0.5 \pm 0.1$ (syt-IV), 
A

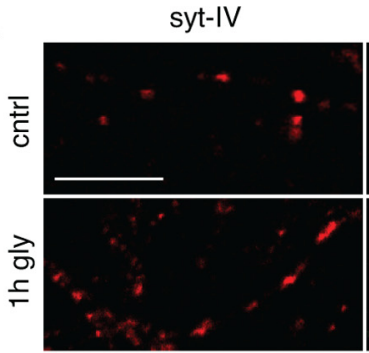

BDNF

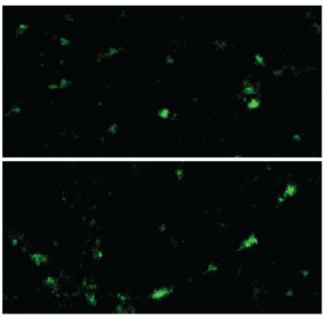

synaptophysin

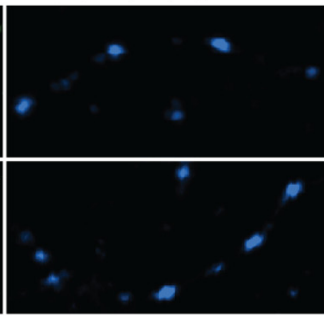

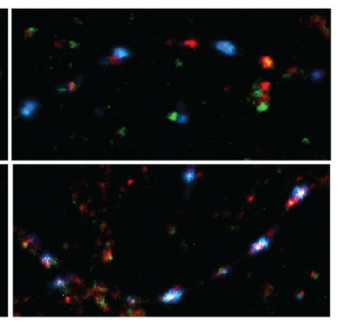

B

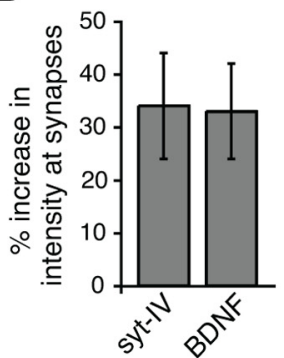

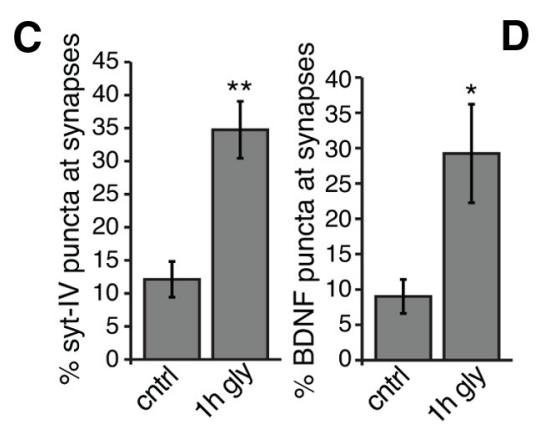
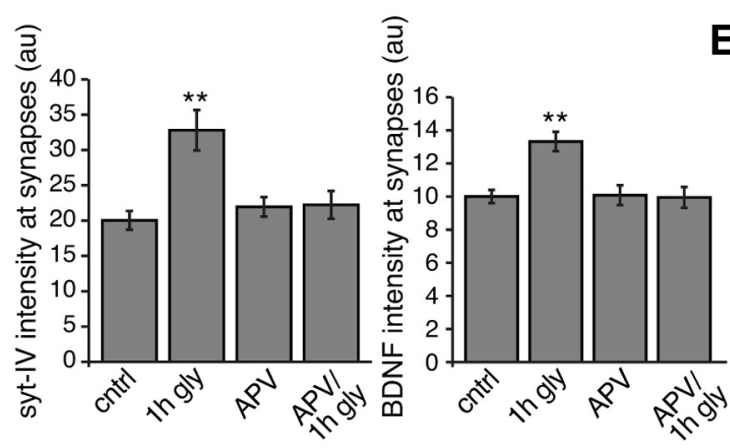

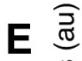

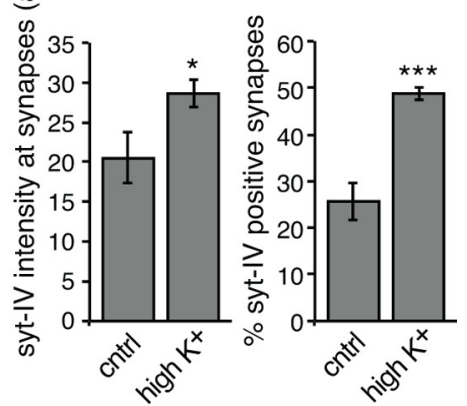

$\mathbf{F}$

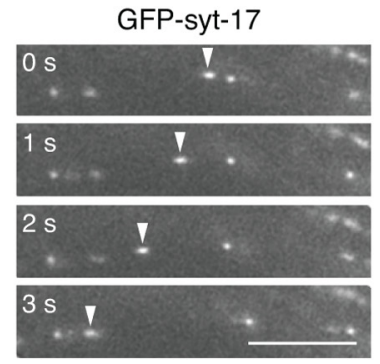

G

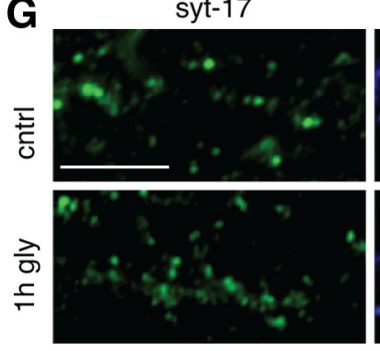

synaptophysin

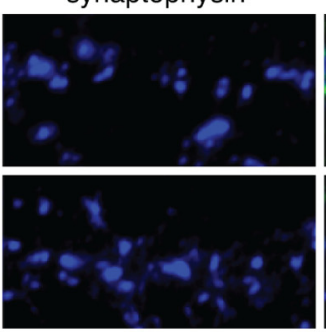

merge

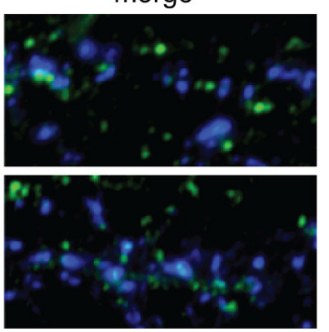

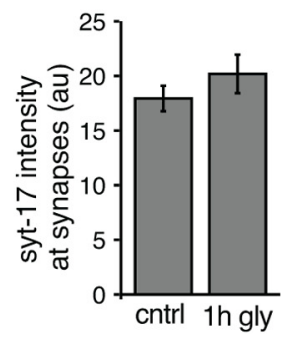

Figure 6. BDNF is recruited to synapses by activity concomitantly with syt-IV. A, Hippocampal cultures immunostained for syt-IV, BDNF, and synaptophysin to mark synapses following treatment with $200 \mu \mathrm{m}$ glycine (gly) for1 h; cntrl, control. B, Quantitation of the intensity of syt-IV and BDNF at synapses marked by synaptophysin. Synaptophysin was thresholded to mark all visible puncta, and the average intensity of syt-IV or BDNF fluorescence within these puncta was calculated and expressed as percentage of control. Pearson's $r$ for syt-IV/synaptophysin $=0.05 \pm 0.06$ (cntrl), $0.22 \pm 0.08$ ( $1 \mathrm{~h} \mathrm{gly})$, and Pearson's $r$ for BDNF/synaptophysin $=0.02 \pm 0.06$ (cntrl l), $0.21 \pm 0.04$ ( $1 \mathrm{~h} \mathrm{gly})(n=10$ images; 2 coverslips $/ 5$ cultures; error bars indicate SEM). C, Quantitation of the percentage of syt-IV and BDNF puncta at synaptic sites in control and glycine-treated cultures ( $n=9$ images; 3 coverslips $/ 3$ cultures; error bars indicate SEM). $D$, Quantitation of syt-IV and BDNF average intensity at synapses marked by synaptophysin in control and glycine, APV, or glycine/APV-treated cultures ( $n=9$ images; 3 coverslips/3 cultures; error bars indicate SEM). E, Quantitation of syt-IV average intensity at synapses marked by synaptophysin and percent syt-IV-positive synapses in control and $45 \mathrm{~mm}$ KCl-treated cultures $(n=9$ images; 3 coverslips 33 cultures; error bars indicate SEM). $\boldsymbol{F}$, Time lapse images of GFP-syt-17 (arrowheads indicate a mobile vesicle transiting along an axonal process). $\mathbf{G}$, Representative images and quantitation of syt-17 average intensity at synapses in control and glycine-treated cultures. Pearson's r for syt-17/synaptophysin $=0.09 \pm 0.03$ (cntrl), $0.07 \pm 0.03$ ( $1 \mathrm{~h}$ gly) $(n=9$ images; 3 coverslips $/ 3$ cultures; error bars indicate SEM). ${ }^{*} p<0.05,{ }^{* *} p<0.01$. Scale bars are $10 \mu \mathrm{m}$ in all panels.

$0.9 \pm 0.3$ (syt-IV + glycine), $0.5 \pm 0.2$ (BDNF), $0.7 \pm 0.2$ (BDNF + glycine). However, following photoactivation of cell bodies after glycine treatment, we observed an accumulation of photoactivated BDNF-PAGFP at "hotspots" along axons and dendrites (Fig. $4 B$ ). We found, using retrospective immunostaining, that these hotspots correspond to synaptic sites (Fig. $4 C$, arrowheads). Following photoactivation, BDNF-PAGFP increased first at proximal synaptic sites and later at more distal sites (Fig. 4D) and accumulated at synapses in greater amounts in glycine-treated neurons compared to controls; the intensity of BDNF-GFP overlapping with synaptophysin was $17.6 \pm 1.7$ (control) and $23.3 \pm 2.6$ (glycine-treated), $p=0.033$.

We next tested the effects of increased neuronal activity on the localization of endogenous syt-IV and BDNF. Treatment of hippocampal neurons with $200 \mu \mathrm{M}$ glycine for $15 \mathrm{~min}$ or $1 \mathrm{~h}$ induced the recruitment of syt-IV to both excitatory and inhibitory synapses, with a slightly greater increase at inhibitory synapses (Fig. $5 A-C)$. VGluT, VGAT, and synaptophysin puncta area was unchanged by glycine treatment and thus could be used to quantify syt-IV accumulation at synapses (VGluT: control $=8.2 \pm 0.8,15$ min glycine $=8.6 \pm 1.4,1 \mathrm{~h}$ glycine $=8.7 \pm 0.8$, forskolin $=$ $8.2 \pm 0.9$, MG132 = 8.6 \pm 1.1 ; VGAT: control $=9.8 \pm 0.7,15$ min glycine $=10.2 \pm 0.9,1 \mathrm{~h}$ glycine $=9.6 \pm 0.7$, forskolin $=$ $10.1 \pm 0.8$, MG132 = 10.4 \pm 0.9 ; synaptophysin: control $=9.3 \pm$ $0.5,1$ h glycine $=9.2 \pm 0.5)$. The average intensity of the syt-IV signal at synapses increased following glycine treatment (Fig. 5F). However, the intensity did not increase uniformly at all synapses in response to activity. Instead, syt-IV was present at relatively high levels at a small subset of synapses in control conditions (Fig. $5 A$, arrow) and was recruited to additional synapses by activity. Therefore, we also assessed the fraction of syt-IV-positive synapses. This fraction increased significantly following glycine treatment (Fig. 5G). Syt-IV may therefore fill a subset of synaptic sites under normal conditions and be recruited to fill additional sites during activity.

Treatment with glycine for $15 \mathrm{~min}$ or $1 \mathrm{~h}$ resulted in the recruitment of syt-IV and BDNF-harboring vesicles to synapses but did not increase overall syt-IV protein levels (Fig. 5F, left inset), suggesting a rapid translocation of pre-existing syt-IV to synaptic sites. In contrast, $4 \mathrm{~h}$ treatment with forskolin increased accumu- 
lation of syt-IV at synapses (Fig. $5 D-G$ ) but also increased syt-IV protein levels (Ibata et al., 2000) (Fig. 5F, right inset). Since syt-IV is rapidly degraded with a half-life of 2 h (Ferguson et al., 1999), we also tested the effects of proteasome inhibition on syt-IV. Treatment with the proteasome inhibitor MG132 resulted in a dramatic increase in syt-IV at synapses and in syt-IV protein levels (Fig. $5 E-G$ ), suggesting that proteasome-mediated degradation may play a role in regulating the level of syt-IV in neurons.

BDNF increased at synapses concomitantly with syt-IV following induction of activity with glycine (Fig. $6 \mathrm{~A}$ ) in terms of both intensity (Fig. $6 B$ ) and percentage of puncta at synapses (Fig. 6C). Blockade of NMDA receptors with $10 \mu \mathrm{M}$ APV 20 min before and during glycine treatment abolished the recruitment of syt-IV and BDNF to synapses (Fig. 6D). Treatment of cultures with $45 \mathrm{~mm} \mathrm{KCl}$ for $10 \mathrm{~min}$ also induced a recruitment of syt-IV to synapses (Fig. 6E). The effects of glycine were specific to syt-IV - and BDNF-containing vesicles, since syt-17 vesicles, which traffic constantly and rapidly in neuronal processes (Fig. $6 F$ ), were not recruited to synapses by glycine treatment (Fig. 6G).

Whether syt-IV itself is localized to synaptic vesicles (Osborne et al., 1999, Ting et al., 2006) or not (Berton et al., 2000, Ibata et al., 2002, Dean et al., 2009) has been an ongoing subject of debate. Although our previous data suggest otherwise (Dean et al., 2009), it remains possible that the syt-IV that is recruited to synapses by activity is localized to synaptic vesicles and not to separate neurotrophincontaining vesicles. To distinguish between these two possibilities we used STED microscopy (Willig et al., 2006) with $40-50 \mathrm{~nm}$ resolution, which allows resolution of single vesicles (Fig. 7A). Using dual color STED, we found that following glycine treatment for $15 \mathrm{~min}$, the syt-IV signal present at synapses was indeed on vesicles that are distinct from synaptophysin-labeled synaptic vesicles (Fig. $7 \mathrm{~B}, C$ ). This contrasts STED images of neurons coimmunostained with synaptophysin and syt-1 (Fig. 7D), where these two proteins largely colocalize on synaptic vesicles. BDNF colocalized with a subset of syt-IV-positive puncta (Fig. $7 E$ ). The absence of BDNF on some syt-IV vesicles could reflect either an inability to detect low levels BDNF or the absence of BDNF on these vesicles, perhaps due to prior release of BDNF cargo (Dean et al., 2009).

Syt-IV and BDNF-harboring vesicles are recruited to both presynaptic and postsynaptic sites

The site of BDNF release and action is an ongoing subject of debate (Kovalchuk et al., 2002, Zakharenko et al., 2003, Gartner et al., 2006), with recent evidence indicating both that postsyn- aptic release affects presynaptic function (Jakawich et al., 2010) and that presynaptic release affects postsynaptic function (Amaral and Pozzo-Miller, 2007, Jia et al., 2010, Li et al., 2010). To determine whether syt-IV is recruited to synapses from the presynaptic or postsynaptic side, i.e., from axonal or dendritic vesicle pools, we made use of a system in which hippocampal neurons are co-cultured with HEK cells expressing the presynaptic adhesion molecule neurexin (Nrx) or its postsynaptic binding partner, neuroligin (Nlg). Nlg-expressing HEK cells, when added to hippocampal cultures and incubated for $24-48 \mathrm{~h}$, induced the formation of presynaptic terminals, marked by the integral synaptic vesicle protein synaptophysin, in contacting axons (Fig. 8A) as shown previously (Scheiffele et al., 2000, Dean et al., 2003). These terminals were not apposed to postsynaptic compartments, as is evident from the lack of GluR2 signal. GFP- 

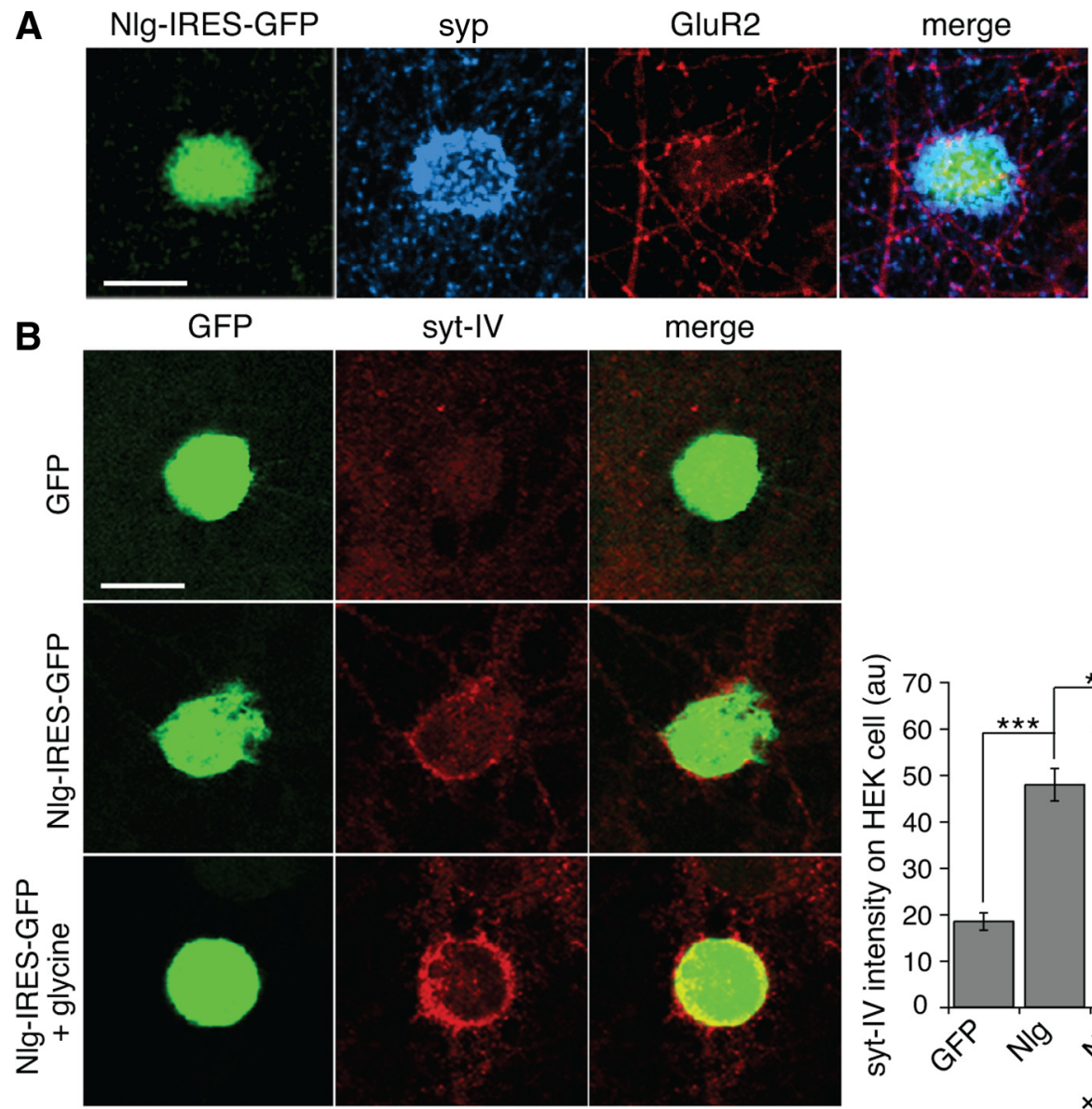

syt-IV

merge
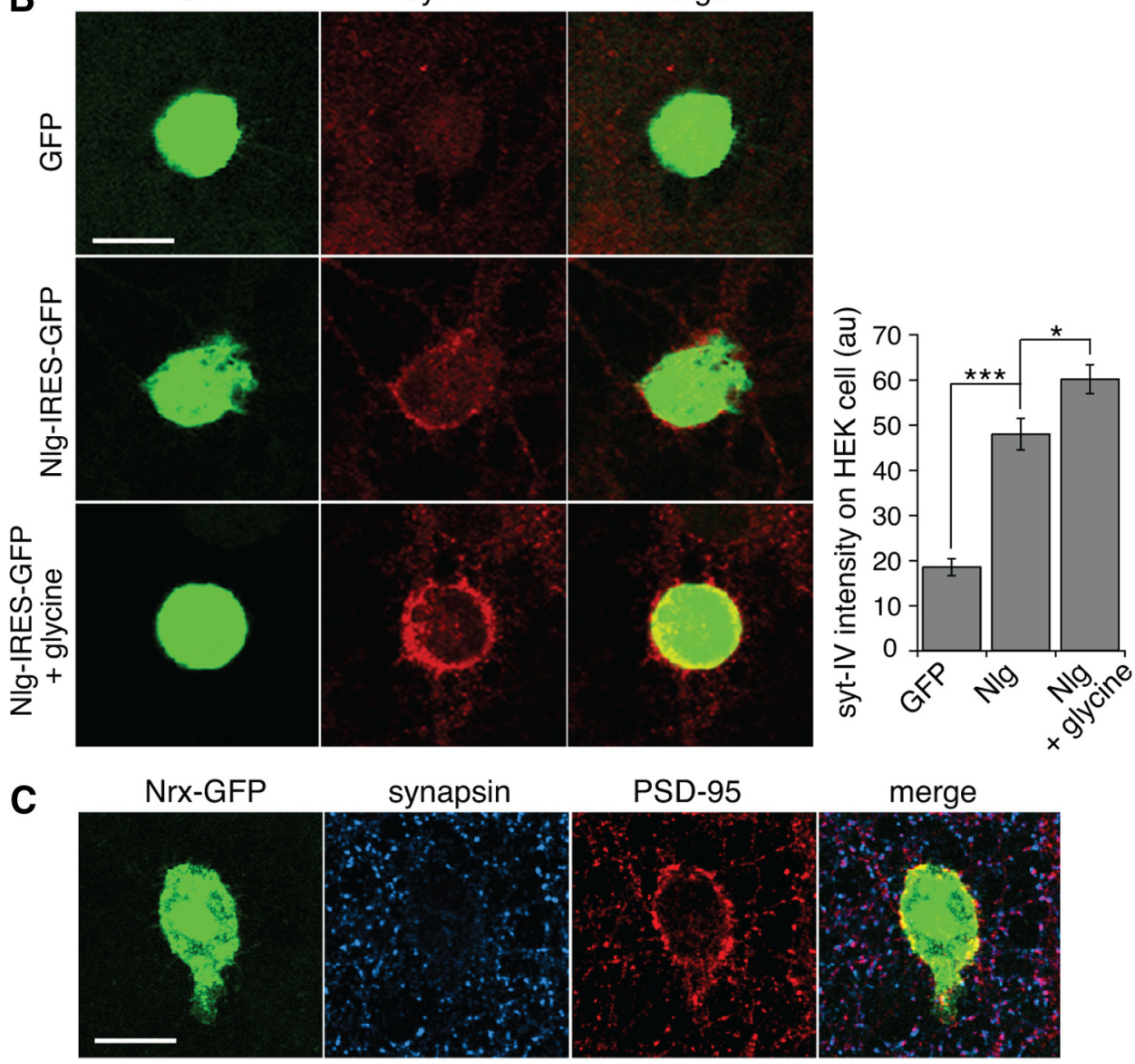

synapsin

PSD-95

merge
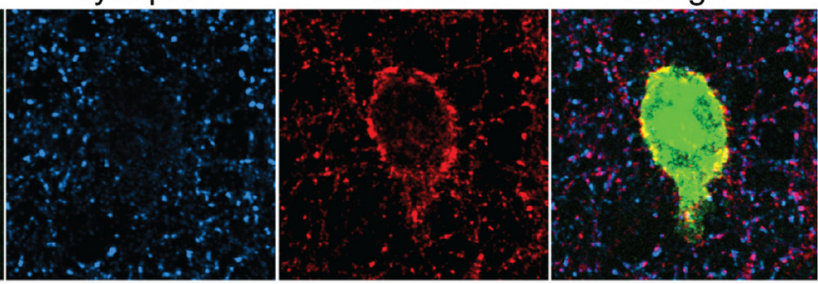

D
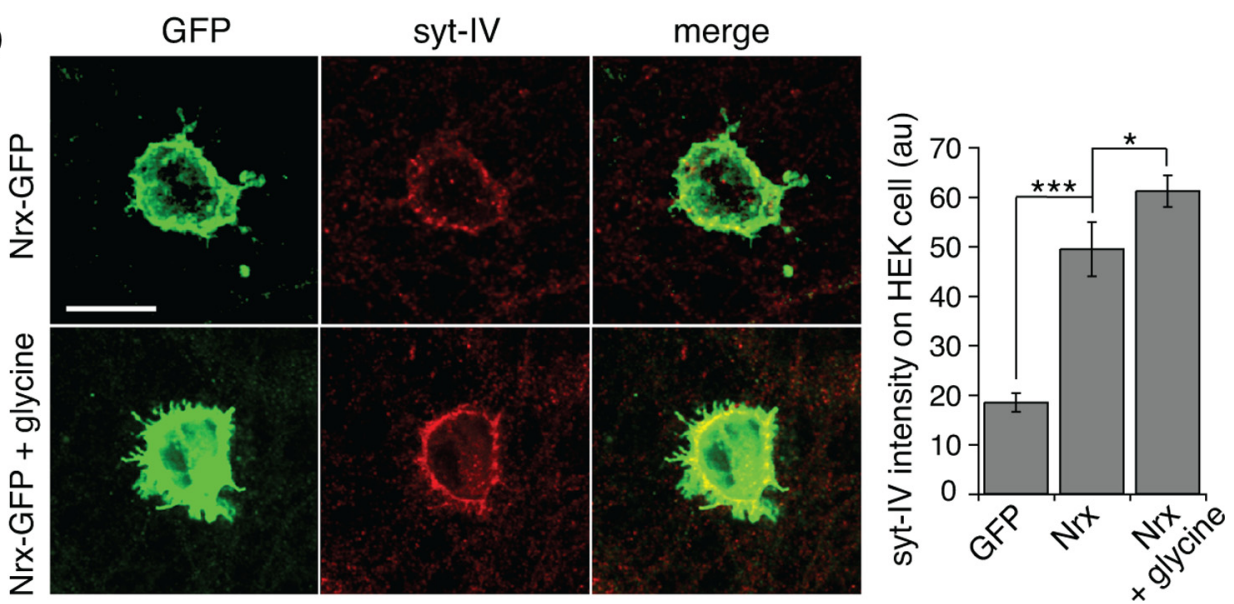

Figure 8. Syt-IV is recruited to presynaptic and postsynaptic sites. A, Neuroligin (Nlg)-expressing HEK cells immunostained for synaptophysin to mark presynaptic terminals and for GluR2 to mark postsynaptic sites. Nlg induced the formation of presynaptic terminals in contacting axons positive for synaptophysin that do not have corresponding postsynaptic structures and thus show no accumulation of GluR2 at the surface of the NIg-expressing HEK cell. B, There was no accumulation of syt-IV signal on GFP-transfected HEK cells (top). In contrast, a ring of syt-IV in presynaptic terminals surrounded NIg-IRES-GFP transfected cells (middle). Treatment with $200 \mu \mathrm{m}$ glycine for $1 \mathrm{~h}$ induced the recruitment of syt-IV to these presynaptic terminals (bottom). $C$, Neurexin (Nrx)-expressing HEK cells immunostained with synapsin to mark presynaptic terminals and PSD-95 to mark postsynaptic sites. Nrx induced postsynaptic "hemisynapses" containing PSD-95 but lacking presynaptic terminals, marked with synapsin. D, A ring of syt-IV surrounding Nrx-GFP transfected HEK cells at postsynaptic sites was evident (top), and this syt-IV signal increased following induction of activity with glycine (bottom) ( $n=20$ cells for each condition; 3 cocultures/transfections; error bars indicate SEM. Significance was determined by a Student's $t$ test; ${ }^{*} p<0.05,{ }^{* *} p<0.01$, and ${ }^{* * *} p<0.001$ ). Scale bars are $10 \mu \mathrm{m}$. 
transfected HEK cells did not induce a significant syt-IV signal in contacting axons, while a ring of syt-IV was clearly present in presynaptic boutons contacting Nlg-expressing cells (Fig. $8 \mathrm{~B}$ ). Following induction of activity for $1 \mathrm{~h}$ with $200 \mu \mathrm{M}$ glycine, the syt-IV signal in these boutons increased in intensity. Since HEK cells have no NMDA receptors, glycine must exert its effects on the neurons in these cocultures, driving the recruitment of syt-IV to presynaptic boutons that contact the neuroligin-expressing HEK cells.

We next tested the converse experiment. Neurexin is the presynaptic receptor of neuroligin and can induce recruitment of postsynaptic proteins to sites of adhesion with contacting dendrites (Graf et al., 2004). When we co-cultured Nrx-expressing HEK cells with hippocampal neurons, we observed a ring of postsynaptic sites, marked by PSD-95, in contacting dendrites that lacked presynaptic terminals, marked by synapsin (Fig. 8C). A ring of syt-IV was observed in postsynaptic sites contacting Nrxexpressing cells (Fig. 8D). This syt-IV signal increased following induction of activity with glycine.

The intensity of bassoon, a presynaptic cytoskeletal active zone protein, contacting Nlg-expressing HEK cells was unchanged by glycine treatment (Fig. 9A), indicating that the presynaptic increase in syt-IV was not due to an increase in synapse formation potentially caused by glycine. Similarly, the intensity of PSD-95 contacting Nrx-expressing HEK cells was also unchanged by glycine treatment (Fig. $9 B$ ), indicating that the increase in syt-IV was due to recruitment to existing postsynaptic sites and not to an increase in synapse number.

As expected from the syt-IV immunofluorescence, BDNF was also present at both presynaptic and postsynaptic sites in a ring surrounding both Nlg-GFP- and Nrx-GFPexpressing HEK cells. This BDNF signal increased in response to activity induced by glycine (Fig. 9C).

Syt-IV is necessary for presynaptic and postsynaptic scaling of

synaptic strength

The recruitment of syt-IV- and BDNFharboring vesicles to synaptic sites in response to neuronal activity (Fig. 5, 6) prompts the hypothesis that the synaptic recruitment of syt-IVharboring BDNF vesicles may act as a homeostatic mechanism to scale synaptic strength (via syt-IV-regulated BDNF release) in response to changes in network activity. If this were the case, syt-IV would not only be upregulated by long-term increases in network activity (such as forskolin treatment for $4 \mathrm{~h}$ ), but also downregulated by decreases in network activity. Blockade of ac$200 \mu \mathrm{m}$ glycine for $1 \mathrm{~h}$.
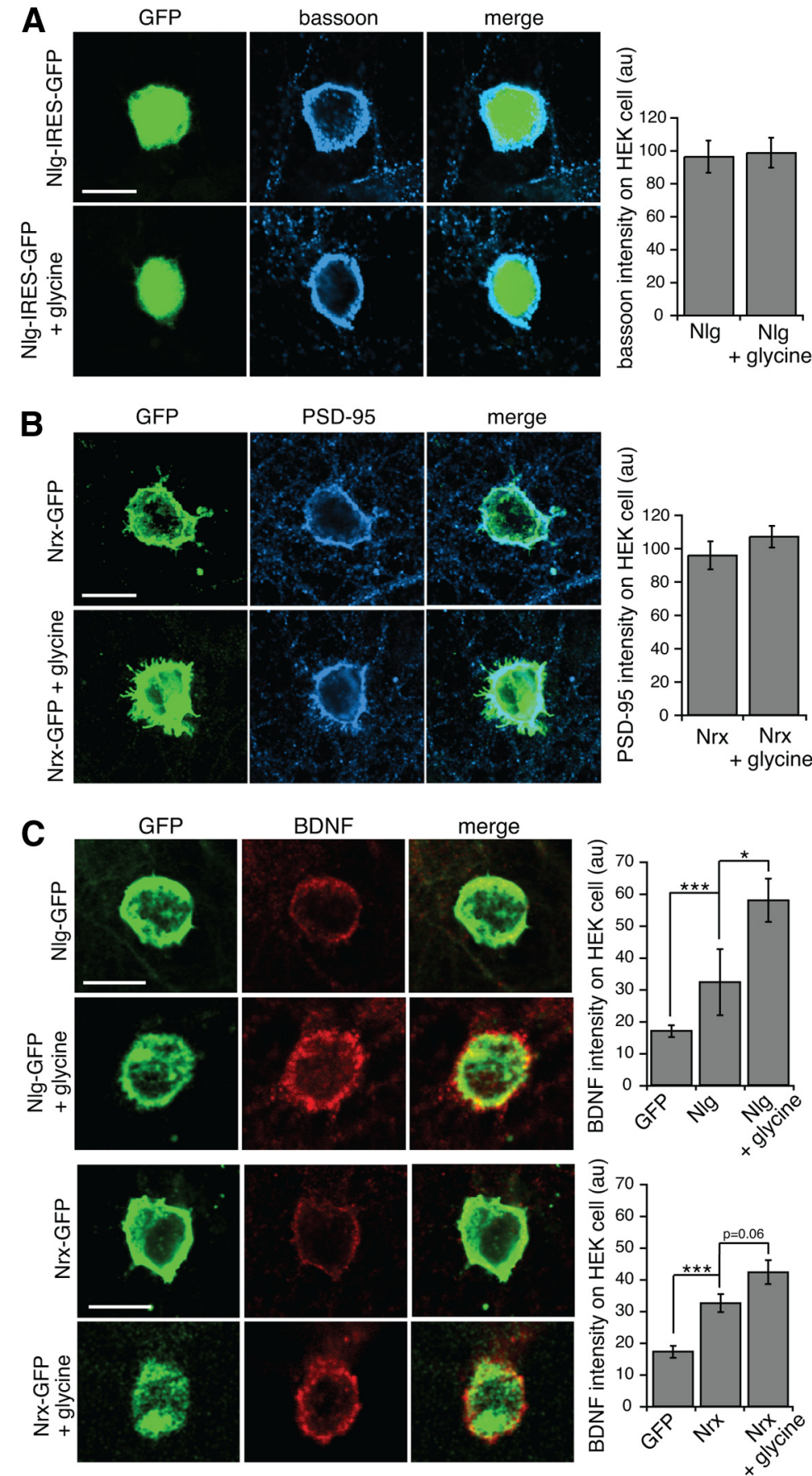

Figure 9. BDNF is recruited concomitantly with syt-IV to pre-existing presynaptic and postsynaptic sites. $\boldsymbol{A}$, Images of $\mathrm{NIg}$ expressing HEK cells co-cultured with hippocampal neurons in control and glycine-treated conditions and immunostained for the presynaptic cytoskeletal active zone marker bassoon. $\boldsymbol{B}$, Images of Nrx-GFP-expressing HEK cells co-cultured with hippocampal neurons in control and glycine-treated conditions and immunostained for the postsynaptic density marker PSD-95. C, A ring of a BDNF signal is observed in presynaptic terminals surrounding NIg-GFP-expressing HEK cells (top) and at postsynaptic sites surrounding Nrx-GFP-expressing HEK cells (bottom). This BDNF signal increases significantly in presynaptic terminals (quantified at top right) and increases slightly (but not significantly) at postsynaptic sites (quantified at bottom right) following treatment with tivity with TTX for $24-48 \mathrm{~h}$ indeed resulted in a reduction in syt-IV protein to barely detectable levels (Fig. 10 A) in comparison to control or to forskolin-treated cultures where syt-IV protein levels increased (Ibata et al., 2000) (Fig. 10A). A decrease in syt-IV expression (and subsequent increase in BDNF release) could act as a "homeostatic" mechanism to increase network excitation by adjusting the strength of synapses to compensate for 


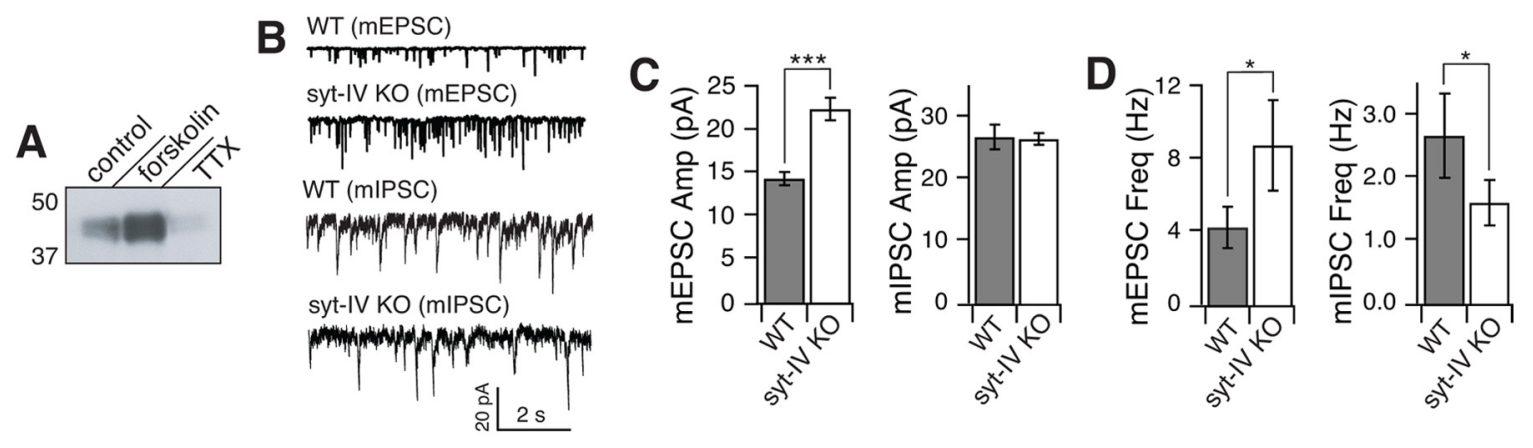

$E$
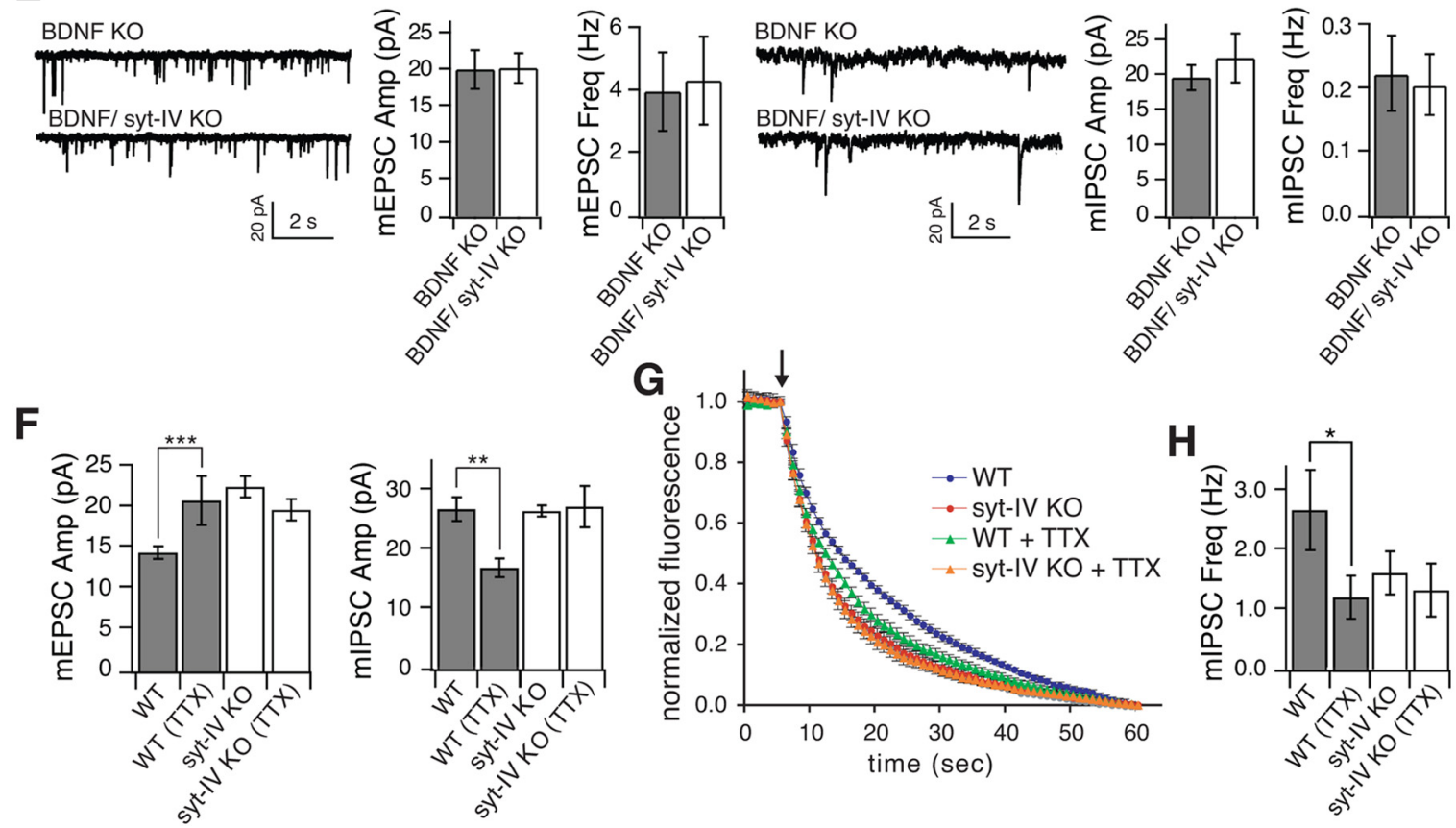

Figure 10. Syt-IV is necessary for scaling of synaptic strength. $\boldsymbol{A}$, Western blot of syt-IV in hippocampal neurons treated with forskolin for $4 \mathrm{~h}$ or TTX for $48 \mathrm{~h}$ compared to control. $\boldsymbol{B}-\boldsymbol{E}$, $\mathrm{mEPSC}$ and mIPSC sample traces $(\boldsymbol{B})$, amplitude (Amp) ( $($ ), and frequency (Freq) (D) from wild-type (WT) and syt-IV knockout (KO) neurons. $\boldsymbol{E}$, $\mathrm{mEPSC}$ and mIPSC amplitude and frequency in BDNF knockout and syt-IV/BDNF double-knockout neurons. $F$, mEPSC and mIPSC amplitude in syt-IV knockout and wild-type neurons treated with TTX for $48 \mathrm{~h}$ compared to control, shown in $\boldsymbol{A}$ (for all mini recordings, $n=7-10$ neurons; $3-4$ coverslips/3-4 cultures; error bars indicate SEM). G, Normalized FM1-43 destaining traces from presynaptic terminals of control and TTX-treated wild-type and syt-IV knockout neurons. $\tau(\mathrm{sec})=17.1 \pm 1.8$ (WT), $9.6 \pm 0.8$ (syt-IV K0), $11.9 \pm 0.8$ (WT + TTX), $8.7 \pm 1.0$ (syt-IV K0 + TTX) $(n=9-15$ coverslips/4 cultures, 15 boutons per coverslip for each condition; error bars indicate SEM). $\boldsymbol{H}$, mIPSC frequency in wild-type and syt-IV knockout neurons in TTX-treated and control conditions ( $n=7-10$ neurons; $3-4$ coverslips/ $3-4$ cultures; error bars indicate SEM).

the global activity blockade induced by TTX (Turrigiano et al., 1998, Burrone and Murthy, 2003, Turrigiano and Nelson, 2004).

If a homeostatic increase in excitation following TTX treatment is caused by downregulation of syt-IV, one would expect that: (1) knockout of syt-IV would mimic the effects of TTXtreatment and increase excitation; and (2) TTX would have no effect on syt-IV knockout neurons where syt-IV is already downregulated, i.e., absent. To test this, we first measured synaptic function in syt-IV knockout neurons. Knockout of syt-IV increased the amplitude and frequency of mEPSCs, as reported previously (Dean et al., 2009), and decreased the frequency of mIPSCs, also as reported previously (Wardle and Poo, 2003), with no change in their amplitude (Fig. $10 B-D$ ). Thus, loss of syt-IV causes a net increase in excitation in terms of mPSCs by affecting both excitatory and inhibitory synapses. If these effects were due to syt-IV acting specifically through BDNF, one would expect no difference in miniature excitatory currents (minis) in syt-IV/BDNF double-knockouts compared to BDNF knockouts (because in both cases there would be no BDNF for syt-IV to act upon). We tested this and found no change in mEPSC or mIPSC amplitude or frequency in syt-IV/BDNF double-knockouts compared to BDNF knockouts (Fig. $10 \mathrm{E}$ ), indicating that syt-IV acts via BDNF. Note that the mIPSC frequency in BDNF knockouts was lower than that in wild type, most likely because BDNF is necessary for the formation and maturation of inhibitory synapses (Abidin et al., 2008, Hong et al., 2008).

We next tested the effects of TTX treatment on wild-type and syt-IV knockout littermate cultures. Activity blockade caused a homeostatic increase in the amplitude of mEPSCs in wild-type cultures as expected (Turrigiano et al., 1998), but it had no effect on syt-IV knockout littermate cultures (Fig. 10F). The amplitude of wild-type mIPSCs was homeostatically decreased by activity blockade, also as expected, but was unchanged in syt-IV knockouts (Fig. $10 F)$. In the case of mIPSCs, syt-IV knockout prevented the reduction in amplitude caused by activity blockade. This observation is consistent with previous findings that excess BDNF (as in syt-IV knockouts (Dean et al., 2009)) prevents the effects of activity deprivation on GABAergic 
A

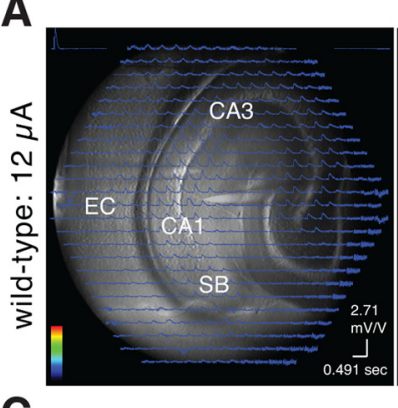

B

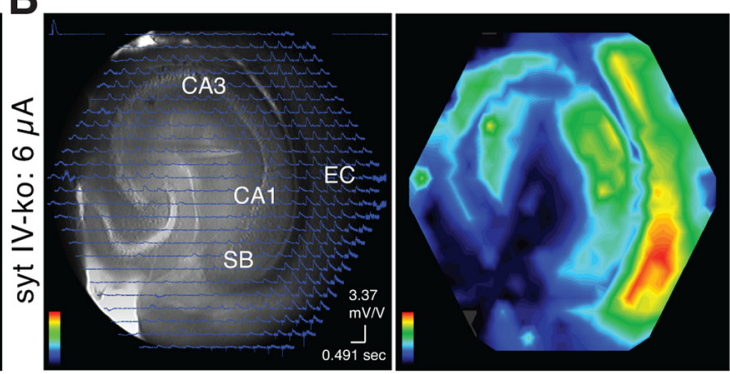

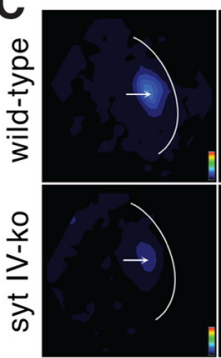

$10 \mathrm{~ms}$

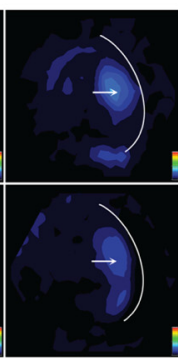

$20 \mathrm{~ms}$

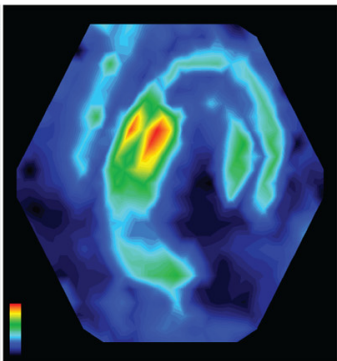

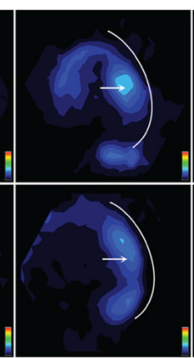

$30 \mathrm{~ms}$

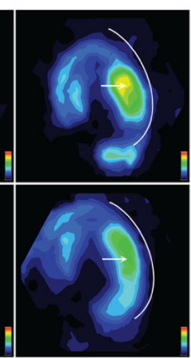

$40 \mathrm{~ms}$

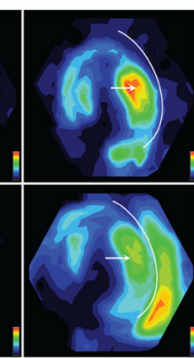

$50 \mathrm{~ms}$

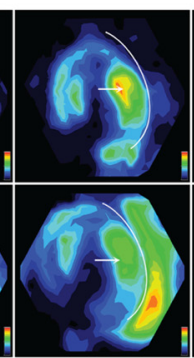

$60 \mathrm{~ms}$

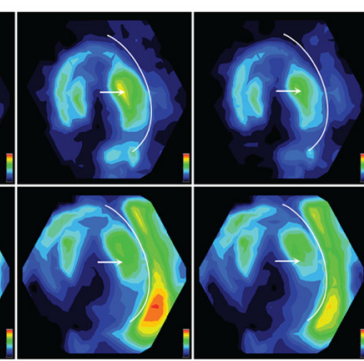

$70 \mathrm{~ms}$

$80 \mathrm{~ms}$

Figure 11. Syt-IV knockouts exhibit enhanced epileptiform responses compared to wild type. $\boldsymbol{A}$, Wild-type hippocampal slice with voltage-sensitive dye optical signals from each of 464 photodiodes overlaid $50 \mathrm{~ms}$ after stimulation with $12 \mu$ A threshold current to induce an epileptiform response. CA1, CA3, subiculum (SB), and entorhinal cortex (EC) are indicated (left). Color-coded amplitude map of the optical signal detected $50 \mathrm{~ms}$ after stimulation and normalized to maximum amplitude is shown on the right. $\boldsymbol{B}$, Syt-IV knockout hippocampal slice with voltage-sensitive dye optical signals overlaid $50 \mathrm{~ms}$ after stimulation with $6 \mu \mathrm{A}$ threshold current to induce an epileptiform response with regions indicated (right) and color-coded amplitude map of the optical signal detected $50 \mathrm{~ms}$ after stimulation, normalized to maximum amplitude (left). C, Time course of a single evoked epileptiform response in a brain slice from wild-type (top) compared to syt IV knockout (bottom) normalized to maximum amplitude for comparison of spatial spread. Border between the hippocampus and entorhinal cortex (white curves), and the location of the stimulating electrode (white arrows) are indicated.

synapses (Rutherford et al., 1997, Swanwick et al., 2006). Thus, loss of syt-IV prevents postsynaptic scaling of synaptic strength.

We additionally tested the effects of chronic increases in activity by treatment with $200 \mu \mathrm{M}$ glycine $/ 10 \mu \mathrm{M}$ bicuculline (Watt et al., 2000, Kennedy et al., 2010) but did not observe a homeostatic response in wild-type neurons with respect to scaling of mIPSC amplitude (wild type $=26.6 \pm 2.0 \mathrm{pA}$, wild type + glycine/bicuculline $=22.2 \pm 1.7 \mathrm{pA}$ ) or frequency (wild type $=$ $2.6 \pm 2.0 \mathrm{~Hz}$, wild type + glycine/bicuculline $=1.0 \pm 0.3 \mathrm{~Hz}$ ), or mEPSC amplitude (wild type $=13.7 \pm 1.2 \mathrm{pA}$, wild type + glycine/ bicuculline $=12.5 \pm 0.8 \mathrm{pA}$ ). We did observe a homeostatic decrease in mEPSC frequency in wild-type neurons following glycine/ bicuculline treatment, and found that syt-IV knockout neurons, by contrast, are unable to scale mEPSC frequency in response to increases in activity (wild type $=4.1 \pm 1.1$, wild type + glycine/ bicuculline $=2.0 \pm 0.5$, syt-IV knockout $=$ $8.7 \pm 2.5$, syt-IV knockout + glycine/ bicuculline $=8.2 \pm 2.3$ ), consistent with the TTX results indicating an inability of syt-IV knockout neurons to scale their strength.

We also tested presynaptic homeostatic scaling in syt-IV knockouts by analyzing FM dye destaining kinetics following activity blockade. Synaptic vesicles were loaded with the lipophilic dye FM1-43 and the kinetics of dye release from presynaptic terminals examined during depolarization with $45 \mathrm{~mm} \mathrm{KCl}$. Chronic blockade of activity in wild-type neurons (before loading with FM1-43) resulted in a homeostatic increase in the rate of FM dye destaining (Wierenga et al., 2006) (Fig. 10G). Syt-IV knockout neurons had a fast FM dye destaining rate to begin with, similar to that of wild-type neurons treated with TTX, and the destaining rate in knockout neurons was unchanged following treatment with TTX. The homeostatic increase in FM dye destaining rate most likely reflects scaling of excitatory synapses, since $\sim 70-80 \%$ of synapses in our hippocampal cultures are excitatory and because the MIPSC frequency in wild-type neurons was homeostatically decreased in response to TTX (Fig. $10 H$ ); in syt-IV knockout neurons, mIPSC frequency was unchanged by TTX treatment.

In summary, activity blockade induced by TTX causes a downregulation of syt-IV, which increases excitation; knockout of syt-IV mimics the homeostatic effect following TTX treatment to increase excitation (via increased BDNF release). In addition, homeostatic responses of mEPSC amplitude, mIPSC amplitude and frequency, and FM dye destaining rate were absent in syt-IV knockout neurons. Thus, the homeostatic response induced by TTX to increase excitation appears to be caused by downregulation of syt-IV to affect BDNF release.

Although EPSCs are unchanged in syt-IV knockout mice compared to wild-type, syt-IV knockouts exhibit an increase in LTP (Ferguson et al., 2004, Dean et al., 2009). This could be due to minis themselves altering the homeostatic threshold for LTP (Sutton et al., 2006), where syt-IV knockout circuits are more "excitable" than wild-type circuits. To test this idea, we examined electrically evoked epileptiform responses in syt-IV knockout and wild-type hippocampal slices using voltage imaging. These evoked discharges show spatiotemporal patterns of spread similar to those reported previously in rat hippocampal slices (Chang et al., 2007). We first determined the threshold to generate an epileptiform response by increasing the stimulus strength in $1 \mu \mathrm{A}$ increments beginning at zero. Syt-IV knockouts had a threshold of $5.4 \pm 0.6 \mu \mathrm{A}$ for epileptiform response, while the threshold for wild-type slices was twice that, $11.5 \pm 0.5 \mu \mathrm{A}(n=5)$. The magnitude of epileptiform response to threshold stimulation was also greater in syt-IV knockouts compared to that in wild type (Fig. $11 A, B$; in wild type, $\Delta I / I=1.49 \pm 0.04 \times 10^{-3}$; in syt-IV knockout, $\left.\Delta I / I=2.10 \pm 0.04 \times 10^{-3}\right)$. In addition, the spatial spread of 
response was larger in syt-IV knockouts, with the response quickly spreading to the entorhinal cortex (Fig. 11C). In contrast, no significant signal was seen in the entorhinal cortex of wildtype slices. This increase in seizure susceptibility in syt-IV knockouts provides an indication that the absence of syt-IV increases the excitability of hippocampal circuits.

\section{Discussion}

The findings reported here lead to three main conclusions: (1) distinct subsets of syt-IV- and BDNF-harboring vesicles are differentially sorted to axons and dendrites and are not shared between these compartments; (2) Syt-IV- and BDNF-harboring vesicles are recruited to both presynaptic and postsynaptic sites in response to glycine-induced increases in neuronal activity; and (3) syt-IV is necessary for both presynaptic and postsynaptic scaling of synaptic strength in response to changes in network activity. Thus, neurons have the ability to dynamically control BDNF release, via regulation by syt-IV, at specific sites along neuronal processes, and to modify synapse and circuit function in response to alterations in network activity.

Apical versus basolateral trafficking has been well described in epithelial cells (Simons and Fuller, 1985, Folsch et al., 1999) where these sorting events are essential for maintaining a selective barrier between the inside and outside of organisms (Cereijido et al., 2004). In neurons, distinct subtypes of syt-IV- and BDNFharboring vesicles may be trafficked to axons versus dendrites to insure that BDNF signaling occurs with specific spatial and temporal dynamics at inputs versus outputs of cells in a network.

Interestingly, BDNF vesicles exhibit distinct modes of depolarization-induced BDNF release assayed by BDNFpHluorin in axons versus dendrites. Dendritic release events are larger and faster than axonal events and are consistent with full fusion and complete release of BDNF-pHluorin (Dean et al., 2009, Matsuda et al., 2009). Syt-IV reduces the magnitude of these events (Dean et al., 2009). In axons, exocytotic events correspond to partial release of BDNF-pHluorin from vesicles, and syt-IV inhibits secretion by increasing the proportion of "nonproductive" fusion events corresponding to transient fusion pore opening without significant release of BDNF. In addition, the intraluminal $\mathrm{pH}$ of syt-IV- and BDNF-harboring vesicles is different in axons and dendrites; dendritic vesicles have a higher $\mathrm{pH}$ than axonal vesicles (Dean et al., 2009).

The segregation of subtypes of syt-IV- and BDNF-harboring vesicles to axons versus dendrites indicates that postsynaptic exocytosis of BDNF might have different effects than presynaptic BDNF release. Indeed, although both presynaptic and postsynaptic BDNF releases affect synaptic function, distinct presynaptic and postsynaptic effects have been found in cocultures of wildtype and syt-IV knockout neurons; postsynaptic release of BDNF, which occurs via full fusion (Dean et al., 2009, Matsuda et al., 2009), increases presynaptic and postsynaptic strength, while presynaptic release, which occurs via partial release of BDNF from vesicles, increases only presynaptic strength (Dean et al., 2009). In addition, postsynaptic release of BDNF is needed to establish the correct ratio of glutamatergic and GABAergic neuron terminals (Singh et al., 2006, Kohara et al., 2007), while presynaptic BDNF, and not postsynaptic BDNF, has been found to be essential for LTP (Zakharenko et al., 2003).

Selective transport via motor proteins that can distinguish axonal versus dendritic microtubules (Nakata and Hirokawa, 2007, Lewis et al., 2009), or exclusion of some syt-IV and BDNF motor/cargo complexes from axons by an axonal selectivity filter (Song et al., 2009) and retention of others, would insure that vesicles with distinct cohorts of proteins that provide specific fusion properties are targeted to axons versus dendrites. Our results provide direct evidence that these axonal and dendritic sytIV- and BDNF-harboring vesicles can be specifically targeted to synapses in response to increased network activity. A highly mobile reservoir of neurotrophin-containing vesicles transiting throughout axons and dendrites may allow for their rapid recruitment to synapses by activity. This phenomenon has been observed at the Drosophila neuromuscular junction, where a mobile pool of peptidergic vesicles are present in axons, and these vesicles are captured at synaptic boutons in response to increases in neuronal activity (Shakiryanova et al., 2006). These observations suggest that neurons can precisely control the site of neurotrophin release and further suggest that this is accomplished via increased capture at active sites, rather than increased synthesis in the soma or increased trafficking of vesicles to distal sites. This would ensure the rapid recruitment of neuropeptide- and neurotrophin-containing vesicles specifically to active sites. Thus, the synaptic recruitment of syt-IV- and BDNF-harboring vesicles and the regulation of BDNF release by syt-IV could potentially modulate synaptic strength on relatively fast time scales in a site-specific manner.

Our data demonstrate that syt-IV affects both presynaptic and postsynaptic function via BDNF and affects excitatory versus inhibitory synapse function differentially, consistent with the effects of exogenous BDNF (Schinder et al., 2000). In addition, we found that syt-IV is necessary for scaling of presynaptic and postsynaptic strength in response to changes in activity. Synaptic scaling has recently been found to occur not only globally but also at specific subcellular sites (Yu and Goda, 2009). Syt-IV is a likely candidate to mediate this local scaling of synaptic strength via regulation of BDNF release.

In summary, while synaptic vesicle exocytosis acts as a fast unidirectional channel of communication between neurons in a network, syt-IV-regulated neurotrophin exocytosis represents a slower bidirectional channel of communication that can be invoked to modulate the first channel in a temporally and spatially specific manner in response to perturbations in activity. This modulation occurs at several levels, including the dynamic regulation of syt-IV expression/ degradation, the incorporation of syt-IV into BDNF vesicles to affect their release, the trafficking of distinct subsets of vesicles with unique fusion properties to axons or dendrites, and the targeting of these vesicles to specific synaptic sites. Thus, BDNF secretion mediated by syt-IV is subject to extensive temporal and spatial control to modulate the spatial pattern of synaptic efficacy in networks of neurons.

\section{References}

Abidin I, Eysel UT, Lessmann V, Mittmann T (2008) Impaired GABAergic inhibition in the visual cortex of brain-derived neurotrophic factor heterozygous knockout mice. J Physiol 586:1885-1901.

Adachi N, Kohara K, Tsumoto T (2005) Difference in trafficking of brainderived neurotrophic factor between axons and dendrites of cortical neurons, revealed by live-cell imaging. BMC Neurosci 6:42.

Amaral MD, Pozzo-Miller L (2007) TRPC3 channels are necessary for brain-derived neurotrophic factor to activate a nonselective cationic current and to induce dendritic spine formation. J Neurosci 27:5179-5189.

Banker GA, Cowan WM (1977) Rat hippocampal neurons in dispersed cell culture. Brain Res 126:397-425.

Berton F, Cornet V, Iborra C, Garrido J, Dargent B, Fukuda M, Seagar M, Marquèze B (2000) Synaptotagmin I and IV define distinct populations of neuronal transport vesicles. Eur J Neurosci 12:1294-1302.

Bloodgood BL, Sabatini BL (2005) Neuronal activity regulates diffusion across the neck of dendritic spines. Science 310:866-869.

Bückers J, Wildanger D, Vicidomini G, Kastrup L, Hell SW (2011) Simulta- 
neous multi-lifetime multi-color STED imaging for colocalization analyses. Opt Express 19:3130-3143.

Burrone J, Murthy VN (2003) Synaptic gain control and homeostasis. Curr Opin Neurobiol 13:560-567.

Cereijido M, Contreras RG, Shoshani L (2004) Cell adhesion, polarity, and epithelia in the dawn of metazoans. Physiol Rev 84:1229-1262.

Chang PY, Jackson MB (2006) Heterogeneous spatial patterns of long-term potentiation in hippocampal slices. J Physiol 576:427-443.

Chang PY, Taylor PE, Jackson MB (2007) Voltage imaging reveals the CA1 region at the CA2 border as a focus for epileptiform discharges and longterm potentiation in hippocampal slices. J Neurophysiol 98:1309-1322.

Chapman ER (2008) How does synaptotagmin trigger neurotransmitter release? Annu Rev Biochem 77:615-641.

Chapman ER, Desai RC, Davis AF, Tornehl CK (1998) Delineation of the oligomerization, AP-2 binding, and synprint binding region of the $\mathrm{C} 2 \mathrm{~B}$ domain of synaptotagmin. J Biol Chem 273:32966-32972.

Dean C, Scholl FG, Choih J, DeMaria S, Berger J, Isacoff E, Scheiffele P (2003) Neurexin mediates the assembly of presynaptic terminals. Nat Neurosci 6:708-716.

Dean C, Liu H, Dunning FM, Chang PY, Jackson MB, Chapman ER (2009) Synaptotagmin-IV modulates synaptic function and long-term potentiation by regulating BDNF release. Nat Neurosci 12:767-776.

Dresbach T, Hempelmann A, Spilker C, tom Dieck S, Altrock WD, Zuschratter W, Garner CC, Gundelfinger ED (2003) Functional regions of the presynaptic cytomatrix protein bassoon: significance for synaptic targeting and cytomatrix anchoring. Mol Cell Neurosci 23:279-291.

Ferguson GD, Thomas DM, Elferink LA, Herschman HR (1999) Synthesis degradation, and subcellular localization of synaptotagmin IV, a neuronal immediate early gene product. J Neurochem 72:1821-1831.

Ferguson GD, Anagnostaras SG, Silva AJ, Herschman HR (2000) Deficits in memory and motor performance in synaptotagmin IV mutant mice. Proc Natl Acad Sci U S A 97:5598-5603.

Ferguson GD, Wang H, Herschman HR, Storm DR (2004) Altered hippocampal short-term plasticity and associative memory in synaptotagmin IV (-/-) mice. Hippocampus 14:964-974.

Fölsch H, Ohno H, Bonifacino JS, Mellman I (1999) A novel clathrin adaptor complex mediates basolateral targeting in polarized epithelial cells. Cell 99:189-198.

Gärtner A, Polnau DG, Staiger V, Sciarretta C, Minichiello L, Thoenen H, Bonhoeffer T, Korte M (2006) Hippocampal long-term potentiation is supported by presynaptic and postsynaptic tyrosine receptor kinase B-mediated phospholipase $\mathrm{C} \gamma$ signaling. J Neurosci 26:3496-3504.

Graf ER, Zhang X, Jin SX, Linhoff MW, Craig AM (2004) Neurexins induce differentiation of GABA and glutamate postsynaptic specializations via neuroligins. Cell 119:1013-1026.

Hong EJ, McCord AE, Greenberg ME (2008) A biological function for the neuronal activity-dependent component of Bdnf transcription in the development of cortical inhibition. Neuron 60:610-624.

Horch HW, Katz LC (2002) BDNF release from single cells elicits local dendritic growth in nearby neurons. Nat Neurosci 5:1177-1184.

Ibata K, Fukuda M, Hamada T, Kabayama H, Mikoshiba K (2000) Synaptotagmin IV is present at the Golgi and distal parts of neurites. J Neurochem 74:518-526.

Ibata K, Hashikawa T, Tsuboi T, Terakawa S, Liang F, Mizutani A, Fukuda M, Mikoshiba K (2002) Non-polarized distribution of synaptotagmin IV in neurons: evidence that synaptotagmin IV is not a synaptic vesicle protein. Neurosci Res 43:401-406.

Jakawich SK, Nasser HB, Strong MJ, McCartney AJ, Perez AS, Rakesh N, Carruthers CJ, Sutton MA (2010) Local presynaptic activity gates homeostatic changes in presynaptic function driven by dendritic BDNF synthesis. Neuron 68:1143-1158.

Jia Y, Gall CM, Lynch G (2010) Presynaptic BDNF promotes postsynaptic long-term potentiation in the dorsal striatum. J Neurosci 30:14440-14445.

Kennedy MJ, Davison IG, Robinson CG, Ehlers MD (2010) Syntaxin-4 defines a domain for activity-dependent exocytosis in dendritic spines. Cell 141:524-535.

Kohara K, Kitamura A, Morishima M, Tsumoto T (2001) Activitydependent transfer of brain-derived neurotrophic factor to postsynaptic neurons. Science 291:2419-2423.

Kohara K, Yasuda H, Huang Y, Adachi N, Sohya K, Tsumoto T (2007) A local reduction in cortical GABAergic synapses after a loss of endogenous brain-derived neurotrophic factor, as revealed by single-cell gene knockout method. J Neurosci 27:7234-7244.

Koh TW, Bellen HJ (2003) Synaptotagmin I, a $\mathrm{Ca}^{2+}$ sensor for neurotransmitter release. Trends Neurosci 26:413-422.

Korte M, Carroll P, Wolf E, Brem G, Thoenen H, Bonhoeffer T (1995) Hippocampal long-term potentiation is impaired in mice lacking brainderived neurotrophic factor. Proc Natl Acad Sci U S A 92:8856-8860.

Kovalchuk Y, Hanse E, Kafitz KW, Konnerth A (2002) Postsynaptic induction of BDNF-mediated long-term potentiation. Science 295:1729-1734.

Kuczewski N, Porcher C, Ferrand N, Fiorentino H, Pellegrino C, Kolarow R, Lessmann V, Medina I, Gaiarsa JL (2008) Backpropagating action potentials trigger dendritic release of BDNF during spontaneous network activity. J Neurosci 28:7013-7023.

Lewin GR, Barde YA (1996) Physiology of the neurotrophins. Annu Rev Neurosci 19:289-317.

Lewis TL Jr, Mao T, Svoboda K, Arnold DB (2009) Myosin-dependent targeting of transmembrane proteins to neuronal dendrites. Nat Neurosci 12:568-576.

Li Y, Calfa G, Inoue T, Amaral MD, Pozzo-Miller L (2010) Activitydependent release of endogenous BDNF from mossy fibers evokes a TRPC3 current and $\mathrm{Ca}^{2+}$ elevations in CA3 pyramidal neurons. J Neurophysiol 103:2846-2856.

Linnarsson S, Björklund A, Ernfors P (1997) Learning deficit in BDNF mutant mice. Eur J Neurosci 9:2581-2587.

Liu IY, Lyons WE, Mamounas LA, Thompson RF (2004) Brain-derived neurotrophic factor plays a critical role in contextual fear conditioning. J Neurosci 24:7958-7963.

Lu W, Man H, Ju W, Trimble WS, MacDonald JF, Wang YT (2001) Activation of synaptic NMDA receptors induces membrane insertion of new AMPA receptors and LTP in cultured hippocampal neurons. Neuron 29:243-254.

Lu Y, Christian K, Lu B (2008) BDNF: A key regulator for protein synthesisdependent LTP and long-term memory? Neurobiol Learn Mem 89:312-323.

Matsuda N, Lu H, Fukata Y, Noritake J, Gao H, Mukherjee S, Nemoto T, Fukata M, Poo MM (2009) Differential activity-dependent secretion of brain-derived neurotrophic factor from axon and dendrite. J Neurosci 29:14185-14198.

Musleh W, Bi X, Tocco G, Yaghoubi S, Baudry M (1997) Glycine-induced long-term potentiation is associated with structural and functional modifications of alpha-amino-3-hydroxyl-5-methyl-4-isoxazolepropionic acid receptors. Proc Natl Acad Sci U S A 94:9451-9456.

Nakata T, Hirokawa N (2007) Neuronal polarity and the kinesin superfamily proteins. Sci STKE 2007:pe6.

Osborne SL, Herreros J, Bastiaens PI, Schiavo G (1999) Calcium-dependent oligomerization of synaptotagmins I and II. Synaptotagmins I and II are localized on the same synaptic vesicle and heterodimerize in the presence of calcium. J Biol Chem 274:59-66.

Poo MM (2001) Neurotrophins as synaptic modulators. Nat Rev Neurosci 2:24-32.

Rutherford LC, DeWan A, Lauer HM, Turrigiano GG (1997) Brain-derived neurotrophic factor mediates the activity-dependent regulation of inhibition in neocortical cultures. J Neurosci 17:4527-4535.

Scheiffele P, Fan J, Choih J, Fetter R, Serafini T (2000) Neuroligin expressed in nonneuronal cells triggers presynaptic development in contacting axons. Cell 101:657-669.

Schinder AF, Berninger B, Poo M (2000) Postsynaptic target specificity of neurotrophin-induced presynaptic potentiation. Neuron 25:151-163.

Shakiryanova D, Tully A, Levitan ES (2006) Activity-dependent synaptic capture of transiting peptidergic vesicles. Nat Neurosci 9:896-900.

Simons K, Fuller SD (1985) Cell surface polarity in epithelia. Annu Rev Cell Biol 1:243-288.

Singh B, Henneberger C, Betances D, Arevalo MA, Rodríguez-Tébar A, Meier JC, Grantyn R (2006) Altered balance of glutamatergic/GABAergic synaptic input and associated changes in dendrite morphology after BDNF expression in BDNF-deficient hippocampal neurons. J Neurosci 26:7189-7200.

Song AH, Wang D, Chen G, Li Y, Luo J, Duan S, Poo MM (2009) A selective filter for cytoplasmic transport at the axon initial segment. Cell 136:1148-1160.

Sutton MA, Ito HT, Cressy P, Kempf C, Woo JC, Schuman EM (2006) Min- 
iature neurotransmission stabilizes synaptic function via tonic suppression of local dendritic protein synthesis. Cell 125:785-799.

Swanwick CC, Murthy NR, Kapur J (2006) Activity-dependent scaling of GABAergic synapse strength is regulated by brain-derived neurotrophic factor. Mol Cell Neurosci 31:481-492.

Ting JT, Kelley BG, Sullivan JM (2006) Synaptotagmin IV does not alter excitatory fast synaptic transmission or fusion pore kinetics in mammalian CNS neurons. J Neurosci 26:372-380.

Tucker KL, Meyer M, Barde YA (2001) Neurotrophins are required for nerve growth during development. Nat Neurosci 4:29-37.

Turrigiano GG, Nelson SB (2004) Homeostatic plasticity in the developing nervous system. Nat Rev Neurosci 5:97-107.

Turrigiano GG, Leslie KR, Desai NS, Rutherford LC, Nelson SB (1998) Activity-dependent scaling of quantal amplitude in neocortical neurons. Nature 391:892-896.

Vician L, Lim IK, Ferguson G, Tocco G, Baudry M, Herschman HR (1995) Synaptotagmin IV is an immediate early gene induced by depolarization in PC12 cells and in brain. Proc Natl Acad Sci U S A 92:2164-2168.

Wang Z, Chapman ER (2010) Rat and Drosophila synaptotagmin 4 have opposite effects during SNARE-catalyzed membrane fusion. J Biol Chem 285:30759-30766.
Wardle RA, Poo MM (2003) Brain-derived neurotrophic factor modulation of GABAergic synapses by postsynaptic regulation of chloride transport. J Neurosci 23:8722-8732.

Watt AJ, van Rossum MC, MacLeod KM, Nelson SB, Turrigiano GG (2000) Activity coregulates quantal AMPA and NMDA currents at neocortical synapses. Neuron 26:659-670.

Wierenga CJ, Walsh MF, Turrigiano GG (2006) Temporal regulation of the expression locus of homeostatic plasticity. J Neurophysiol 96:2127-2133.

Willig KI, Rizzoli SO, Westphal V, Jahn R, Hell SW (2006) STED microscopy reveals that synaptotagmin remains clustered after synaptic vesicle exocytosis. Nature 440:935-939.

Yu LM, Goda Y (2009) Dendritic signalling and homeostatic adaptation. Curr Opin Neurobiol 19:327-335.

Zakharenko SS, Patterson SL, Dragatsis I, Zeitlin SO, Siegelbaum SA, Kandel ER, Morozov A (2003) Presynaptic BDNF required for a presynaptic but not postsynaptic component of LTP at hippocampal CA1-CA3 synapses. Neuron 39:975-990.

Zhang X, Poo MM (2002) Localized synaptic potentiation by BDNF requires local protein synthesis in the developing axon. Neuron 36:675688. 